\title{
Thermo-mechanical numerical model of the transition from continental rifting to oceanic spreading: the case study of the Alpine Tethys
}

\author{
ANNA MARIA MAROTTA*, MANUEL RODA, KATYA CONTE \\ \& MARIA IOLE SPALLA
}

Department of Earth Sciences ‘Ardito Desio’, Università degli Studi di Milano, via L. Mangiagalli 34 I-20134, Milan, Italy

(Received 14 June 2016; accepted 29 July 2016; first published online 3 October 2016)

\begin{abstract}
We develop a two-dimensional thermo-mechanical numerical model in which the formation of oceanic crust and serpentinite due to the hydration of the uprising mantle peridotite has been implemented, with the aim of discussing the behaviour of the lithosphere of the Alps and Northern Apennines during the transition from continental rifting to ocean spreading of the Alpine Tethys. The predictions of the model are compared with natural data related to the Permian-Triassic hightemperature - low-pressure (HT-LP) metamorphism affecting the continental lithosphere and data from the Jurassic $P-T$ evolution of the oceanic lithosphere from the Alps and the Northern Apennines. Our analysis indicates that a thinned continental crust, an ocean-continent transition zone and an oceanic lithosphere characterize the final structure of the system in a poor magma rift pre-Alpine configuration. We also find that mantle serpentinization starts before crustal break-up and that denudation occurs before ocean spreading. The mantle denudation starts several million years before the gabbros/basalt formation, generating an ocean-continent transition zone from the passive continental margin to the oceanic lithosphere of size 160-280 km. The comparative analysis shows that the extension of a hot and weak lithosphere, which promotes the development of hyperextended Alpine margins, better agrees with the natural data. Finally, our comparative analysis supports the hypothesis that the lithospheric extension preceding the opening of the Alpine Tethys did not start in a stable continental lithosphere, but developed by recycling part of the old Variscan collisional suture.
\end{abstract}

Keywords: 2D FEM, Alps, Apennines, continental break-up, ocean-continent transition zone.

\section{Introduction}

The aim of the present work is to discuss the thermomechanical behaviour of the lithosphere of the Alps and Northern Apennines during the transition from continental rifting to oceanization of the Alpine Tethys.

After the Variscan Orogeny, the future Alpine area (Fig. 1) underwent an extensional stage leading to the break-up of the Pangaea continental lithosphere and the opening of the Alpine Tethys Ocean (Lardeaux \& Spalla, 1991; Diella, Spalla \& Tunesi, 1992; Dal Piaz, 1993; Bertotti et al. 1993; Handy et al. 1999; Schuster et al. 2001; Schuster \& Stüwe, 2008; Marotta, Spalla \& Gosso, 2009). The influence of the thermal, structural and compositional inheritance of the Variscan collision and collapse on the following extensional stage is still under debate (e.g. Marotta \& Spalla 2007; Von Raumer et al. 2013; Spalla et al. 2014).

The thermal structure of the Alpine lithosphere before the rifting event is poorly understood. Based on petrological analyses of the Ivrea crustal section, Handy et al. (1999) and Smye \& Stockli (2014) proposed that a series of thermal pulses after Carboniferous time affected the pre-rifting Alpine crust, potentially associ-

\footnotetext{
*Author for correspondence: anna.maria.marotta@unimi.it
}

ated with and following the asthenosphere upwelling driven by hyperextension of the Adriatic margin during Late Triassic - Early Jurassic time. According to recent interpretations, the elevated temperatures in the deep crust of the Ivrea Zone may persist for millions of years, remaining close to the solidus for approximately $30 \mathrm{Ma}$ (Klötzli et al. 2014) and thermally perturbing the Alpine lithosphere before the beginning of the rifting. Passive extension in the EuropaAdria system is thought to have been active during Triassic time (Handy \& Zingg, 1991; Muntener, Hermann \& Trommsdorf, 2000; Muntener \& Hermann, 2001; Montanini, Tribuzio \& Anczkiewicz, 2006) or to have started during late Palaeozoic time (Lardeaux \& Spalla, 1991; Diella, Spalla \& Tunesi, 1992; Dal Piaz, 1993; Marotta \& Spalla, 2007; Marotta, Spalla \& Gosso, 2009). In contrast, based on data from Val Malenco, Corsica, Erro-Tobbio and Voltri Massif, Piccardo, Padovano \& Guarnieri (2014) proposed that the pre-rift mantle lithosphere was equilibrated along an intermediate subcontinental geothermal gradient and the Permian high-temperature event(s) was followed by isobaric cooling, lasting more than $50 \mathrm{Ma}$ (Manatschal, 2004). This interpretation suggests the occurrence of a thermally equilibrated lithosphere before the rifting event (Lavier \& Manatschal, 2006). This idea is 


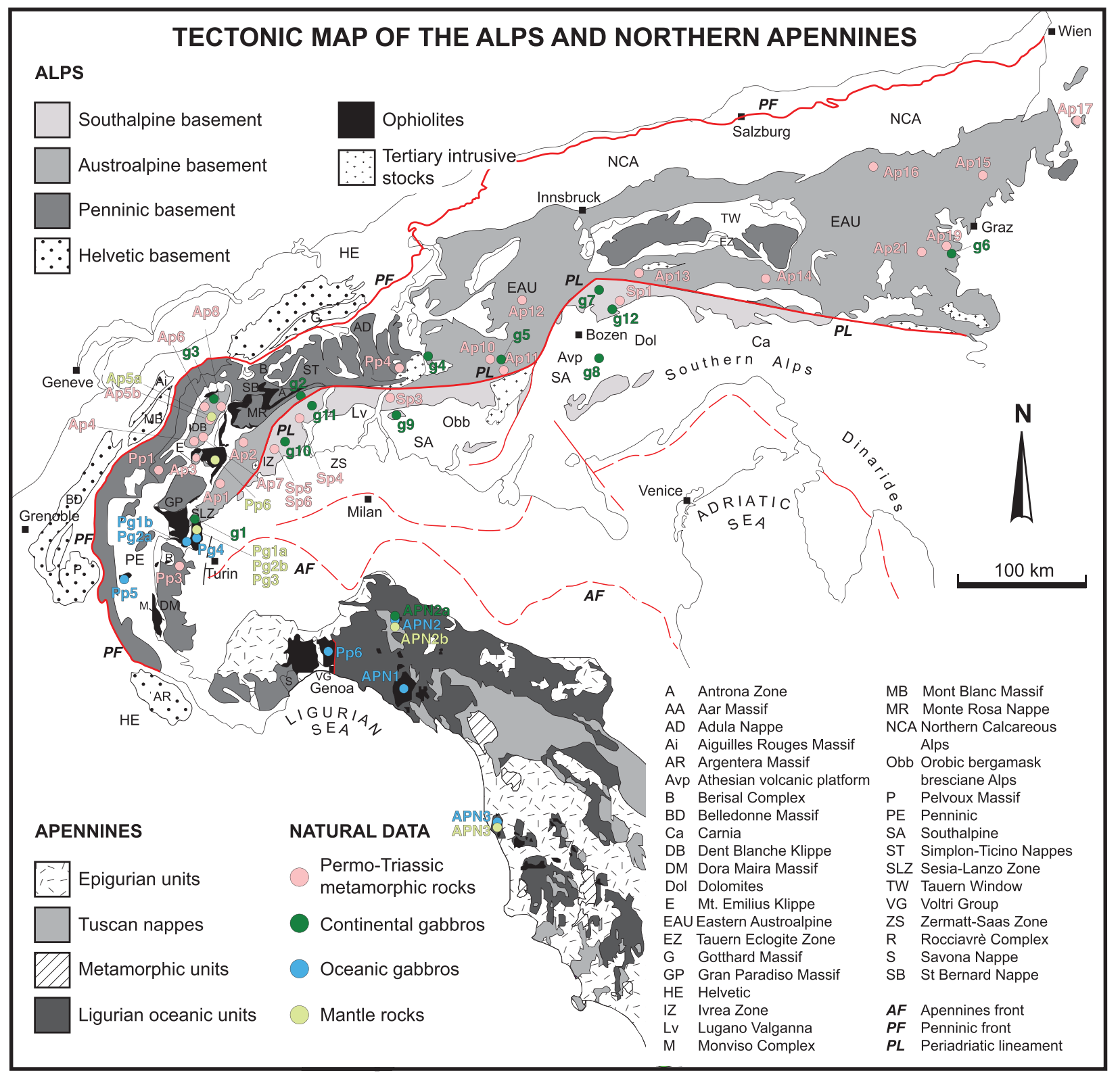

Figure 1. (Colour online) Tectonic map of the Alps and Apennines (after Marotta \& Spalla, 2007; Handy et al. 2010) with the locations of: metamorphic rocks and main gabbro bodies of Permian-Triassic age occurring in the pre-Alpine continental crust of the Alps; and mantle rocks and oceanic gabbros from the Alps and Northern Apennines. The codes are defined in Tables 2-5.

supported by numerous rifting models proposed for the Alpine area (e.g. Beltrando, Rubatto \& Manatschal, 2010; Mohn et al. 2012) that were conceived based exclusively on data from the exploration of the Iberia passive margin (e.g. Boillot, Beslier \& Girardeau, 1995; Hébert et al. 2008).

Numerous numerical and analogue models of continental extension highlight the roles of different parameters and mechanisms in dictating the final geometry and style of the rifting and continental break-up. Among others, Reston \& Morgan (2004), Van Avendonk et al. (2009), Brune \& Autin (2013) and Brune et al. (2014) focus on the role of the thermal state of the lithosphere; Buck (1991), Corti et al. (2004), Nagel \& Buck (2004), Huismans, Buiter \& Beaumont (2005), Huismans \& Beaumont (2011, 2014), Cloetingh et al. (2013), Brune et al. (2014) and Liao \& Gerya (2015) analyse the effects of the composition, rheology and strength of the lower crust; Brune (2014) and Brune et al. (2014) investigate the role of the extensional velocity; Manatschal, Lavier \& Chenin (2015) and Naliboff \& Buiter (2015) examine the role of structural and compositional inheritance of the system; and Escartín, Hirth \& Evans (1997) and Pérez-Gussinyé et al. (2001, 2006) focus on serpentinization. However, these works are not strictly focused on the opening of the Alpine Tethys and do not perform systematic comparisons between the model predictions and the natural data from the continental and oceanic crust of the Alps and Northern Apennines, including $P-T$ estimates, radiometric ages and lithological affinity.

This work represents an advancement of the work of Marotta, Spalla \& Gosso (2009) in which a rifting process following a continental collision and ending before 
the break-up of the continental crust is modelled. They implemented a bi-dimensional numerical geodynamic model to analyse the effects of an active extension during the Permian-Triassic period (300-220 Ma), assuming that the extension developed in a lithosphere already thermally and mechanically perturbed by a previous subduction-collision phase which occurred during the Variscan age, up to 300 Ma. Marotta, Spalla \& Gosso (2009)'s results supported the idea of an asymmetric rifting in which the Adriatic continental crust represented the hanging wall, although satisfactory and complete agreement with the natural geological data in terms of the coincidence of age, lithology and $P-T$ values was only obtained at the higher rate of forced extension $\left(2 \mathrm{~cm} \mathrm{a}^{-1}\right)$.

Indeed, Marotta, Spalla \& Gosso (2009)'s model has two main limits: (a) the model does not evolve until the continental lithosphere break-up and subsequent ocean spreading; and (b) the conditions favourable to mantle partial melting have not been considered; the timing of the beginning of the new oceanic lithosphere was therefore not predicted. For these reasons, in the present work the transition from continental rifting to ocean spreading has been investigated using a two-dimensional (2D) thermo-mechanical numerical model in which the serpentinite formation due to the hydration of the upraising peridotite has been implemented and extension occurs over a mechanically unperturbed lithosphere. The predictions of the model have been compared with natural data related to the Permian-Triassic high-temperature - low-pressure (HT-LP) metamorphism affecting the continental lithosphere and data from the pre-Alpine Jurassic $P-T$ evolution of the oceanic crust from the Alps and the Northern Apennines.

\section{Numerical model}

\section{2.a. Model set-up}

To simulate the transition from rifting to oceanic spreading, a time-dependent 2D thermo-mechanical numerical model has been used in which the dynamics of the crust-mantle system have been investigated by numerical integration of the three fundamental equations of conservation of mass, momentum and energy:

$$
\begin{aligned}
\nabla \cdot \vec{u} & =0 \\
-\nabla P+\nabla \cdot \vec{\tau}+\rho \vec{g} & =0 \\
\rho c_{p}\left(\frac{\partial T}{\partial t}+\vec{u} \cdot \nabla T\right) & =\nabla \cdot(K \nabla T)+\rho H_{d}
\end{aligned}
$$

respectively, where $\vec{u}$ is the velocity, $P$ is the pressure, $\vec{\tau}$ is the deviatoric stress, $\rho$ is the density, $\vec{g}$ is the gravity acceleration, $c_{p}$ is the specific heat at constant pressure, $T$ is the temperature, $K$ is the thermal conductivity and $H_{d}$ is the radiogenic heat production rate per unit mass.

Equations (1), (2) and (3) are solved using the 2D finite-elements code SubMar, which has been exhaustively described by Marotta, Spelta \& Rizzetto (2006).
This numerical code uses the penalty function formulation to integrate the equation for the conservation of momentum and the streamline upwind/Petrov-Galerkin method to integrate the equation for the conservation of energy.

The marker in-cell technique has been used to compositionally differentiate crust and mantle rocks.

A viscous-plastic behaviour has been assumed for both materials. The effective viscosity is calculated as follows:

$$
\mu^{\text {eff }}=\mu_{\text {viscous }}=\mu_{0, i} \exp \left[\frac{E_{i}}{R}\left(\frac{1}{T}-\frac{1}{T_{0}}\right)\right]
$$

where $\mu_{0, i}$ and $E_{i}$ are the reference viscosity at the reference temperature $T_{0}$ and the activation energy for the crust $(i=\mathrm{c})$ and the mantle $(i=\mathrm{m})$, respectively, with a maximum value defined by the plastic viscosity assumed equal to $10^{25} \mathrm{~Pa}$ s (Table 1 ).

We account for a brittle behaviour of the crust to define the rheological conditions for mantle serpentinization only, as better specified in Section 2.b.

The material parameters are listed in Table 1. Initially, the lithosphere is mechanically unperturbed and laterally homogeneous. The initial thickness of the continental crust is assumed to be $30 \mathrm{~km}$ (Fig. 2), which is in agreement with models envisaging the beginning of extension-transtension onto a lithosphere characterized by a crust with a thickness of approximately $30 \mathrm{~km}$ (Muntener, Hermann \& Trommsdorf, 2000; Manatschal, 2004).

Two thermal settings are proposed here to satisfy two contrasting pre-rifting settings of the Alpine lithosphere characterized by different depths of the $1600 \mathrm{~K}$ isotherm: 80 and $220 \mathrm{~km}$ (Fig. 2b). We refer to these simulations as the hot and cold models, respectively. The initial thermal conditions correspond to an almost conductive thermal profile from $300 \mathrm{~K}$ at the surface to $1600 \mathrm{~K}$ at the base of the lithosphere; an initial homogeneous temperature of $1600 \mathrm{~K}$ is assumed below the lithosphere (Fig. 2b).

Boundary conditions are defined in terms of the temperature and velocity. A temperature of $300 \mathrm{~K}$ is fixed at the top of the crust and throughout the air-water layer, a temperature of $1600 \mathrm{~K}$ is fixed at the base of the model and zero flux is assumed through the lateral sides of the model. We apply an extension rate of $1.25 \mathrm{~cm} \mathrm{a}^{-1}$ at both lateral sides of the model throughout the crustal thickness, resulting in a symmetric passive rifting with a total extension rate of $2.5 \mathrm{~cm} \mathrm{a}^{-1}$, compatible with the magma-poor nature of the rift (e.g. Manatschal $\&$ Müntener, 2009). The 2D domain is closed vertically with shear-free conditions prescribed along the top and the bottom of the model domain, while both crust and lithospheric mantle are allowed to exit the model boundaries allowing the thinning of either crust and lithospheric mantle (Fig. 2a).

Considering that in the Alpine literature the onset of rifting is proposed to occur during $220-200 \mathrm{Ma}$ (Müntener, Hermann \& Trommsdorf, 2000; Manatschal, 2004; Montanini, Tribuzio \& Anczkiewicz, 
Table 1. Material properties used in the 2-D numerical modelling

\begin{tabular}{lllll}
\hline & \multicolumn{1}{c}{ Continental crust } & \multicolumn{1}{c}{ Mantle } & Serpentinized mantle & Air or water \\
\hline Composition & $66 \%$ gneiss $+33 \%$ granite & $100 \%$ dunite & $100 \%$ serpentinite & \\
Mean density $\left(\mathrm{kg} \mathrm{m}^{-3}\right)$ & $2640^{\mathrm{a}}$ & $3200^{\mathrm{a}}$ & $3000^{\mathrm{f}}$ & $1180^{\mathrm{a}}$ \\
Radiogenic heat production $\left(10^{-6} \mathrm{~W} \mathrm{~m}^{-3}\right)$ & $2.5^{\mathrm{b}}$ & $0.002^{\mathrm{b}}$ & $0.002^{\mathrm{b}}$ & 0 \\
Thermal conductivity $\left(\mathrm{W} \mathrm{m}^{-1} \mathrm{~K}^{-1}\right)$ & $3.06^{\mathrm{b}}$ & $4.15^{\mathrm{b}}$ & $4.15^{\mathrm{b}}$ & $0.026^{\mathrm{b}}$ \\
Rheology & Dry granite $^{\mathrm{c}}$ & Dry dunite $^{\mathrm{d}}$ & Hydrated dunite & Air or water \\
Activation energy $(\mathrm{kJ} / \mathrm{mol})$ & 123 & 444 & & $10^{20}$ \\
Reference viscosity $(\mathrm{Pa} \mathrm{s})$ & $3.47 \times 10^{21}$ & $5.01 \times 10^{20}$ & $10^{19}$ & \\
Maximum plastic viscosity (Pa s) & $10^{25}$ & $10^{25}$ & & \\
\hline
\end{tabular}

${ }^{\mathrm{a}}$ Dubois \& Diament (1997) and Best \& Christiansen (2001); ${ }^{\mathrm{b}}$ Rybach (1988); ${ }^{\mathrm{c}}$ Ranalli \& Murphy (1987); ${ }^{\mathrm{d}}$ Chopra \& Peterson (1981); ${ }^{\mathrm{e}}$ Honda \& Saito (2003), Arcay, Tric \& Doin (2005) and Roda, Marotta \& Spalla (2010).

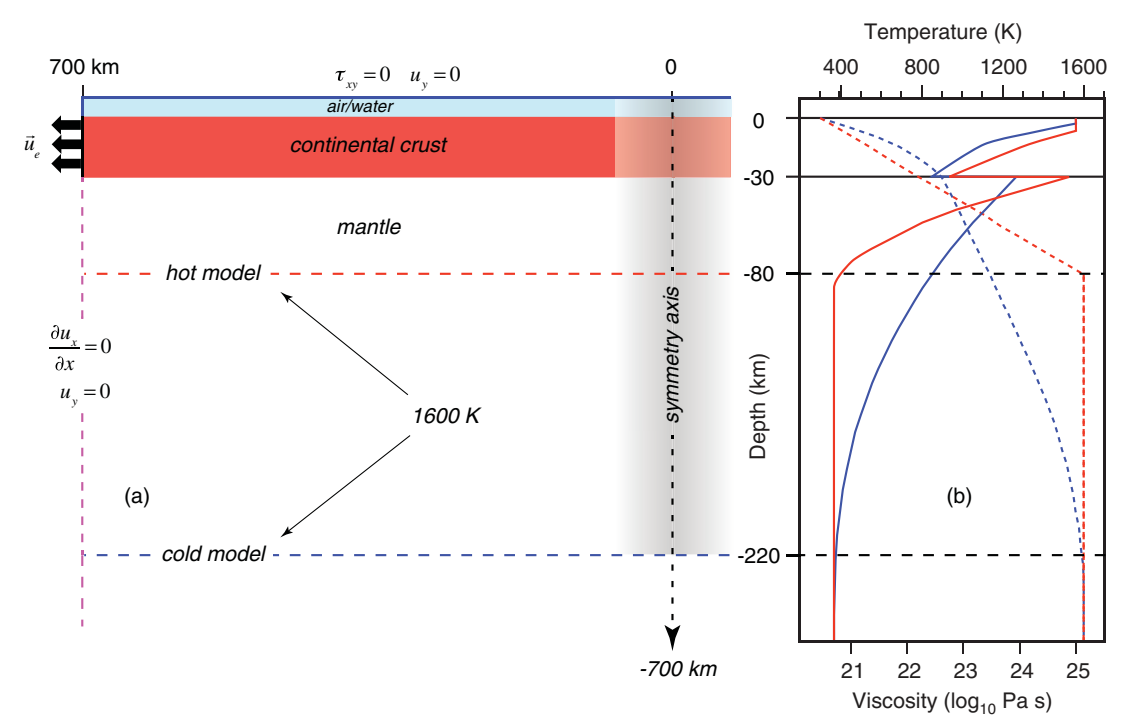

Figure 2. (Colour online) (a) 2D geometry and numerical set-up of the model. (b) Thermal and rheological profiles at the beginning of the evolution for the hot (red lines) and cold (blue lines) models. The solid lines indicate the effective viscosity profiles, corresponding to the geotherms indicated by the dashed lines.

2006; Piccardo, Padovano \& Guarnieri, 2014) and that the oldest rocks of the ophiolitic associations belonging to the Liguria-Piemonte Ocean have been dated at 175-160 Ma (Tribuzio, Thirwall \& Vannucci, 2004; Rossi et al. 2012; Kaczmarek, Müntener \& Rubatto, 2008; Li et al. 2013, 2015), we run the simulation over a time span of $60 \mathrm{Ma}$ to match the time interval needed to generate the oldest gabbro intrusions in the natural system.

\section{2.b. Conditions for mantle serpentinization}

Magmatic-poor margins formed by the rifting of continental crust are characterized by the occurrence of serpentinized peridotites within a broad continent-ocean transition (e.g. Pérez-Gussinyé et al. 2001; Manatschal, 2004) and by the lithostratigraphy of the ophiolitic sequences (Mevel, Caby \& Kienast, 1978; Lagabrielle \& Cannat, 1990; Chalot-Prat, 2005; Manatschal et al. 2011; Li et al. 2013). The role of serpentinization of the lithospheric mantle during continental rifting and the transition to oceanic spreading has been extensively discussed by Pérez-Gussinyé et al. $(2001,2006)$. They assume that mantle serpentinization occurs when the overlaying crustal layer is under brittle conditions, so that faults can cut across the crust allowing hydrous fluids to penetrate the mantle, and mantle matches the appropriate pressure and temperature conditions for the stability field of serpentine.

In order to implement mantle serpentinization and the consequent rheological and compositional changes, we check whether the pressure and temperature of each mantle-type marker match the stability field of serpentine and the overlying crustal layer is under brittle conditions. To define whether the overlying layer is under brittle conditions, we use a simplified formulation of Byerlee's law criterion:

$$
\sigma_{\text {brittle }}=\beta \cdot y \quad \text { with } \quad \beta=16 \mathrm{MPa} \mathrm{km}^{-1}
$$

where $y$ is the depth, and compare the brittle strength $\sigma_{\text {brittle }}$ with the temperature- and pressure-based plastic strength:

$$
\text { Regime } \Leftarrow \min \left\{\sigma_{\text {brittle }}, \sigma_{\text {plastic }}\right\} .
$$

\section{2.c. Conditions for partial melting}

During an active extension of a continental lithosphere, the temperature in the lithospheric mantle increases as 
a consequence of the upwelling asthenospheric flow. If the pressure and temperature conditions of peridotite solidus are matched, partial melting of the lithospheric mantle occurs and gabbroic-basaltic melts form. We assume here that once the mantle partial melting occurs in the system, the oceanic lithosphere starts to form. This implies an instantaneous transfer of the mantle melt to the surface in agreement with the estimates of the rate of magma ascent across the continental and oceanic crust (Clague, 1987; Turner et al. 2000).

In order to individuate the beginning of oceanic spreading and to identify the extension of the partially molten mantle region, we check the pressure and temperature conditions of each mantle-type marker during the evolution. When the $P-T$ conditions reach the dry solidus field of peridotite (Rogers et al. 2008):

$$
P_{\mathrm{m}} \leq \alpha \cdot T_{\mathrm{m}}+\beta,
$$

where $\alpha=0.00789792857 \mathrm{GPa} \mathrm{K}^{-1}$ and $\beta=-$ $11.1071202 \mathrm{GPa}$, its typology is changed from mantle type into potential partially molten mantle type.

The extension of the partially molten mantle region is shown in Figure 3 distinguishing the oceanic lithosphere (dark green), formed by serpentinized mantle hosting gabbros and basalts that can be produced once partial melting conditions are attained, from the mantle that serpentinized before the occurrence of partial melting (light green).

In the present form, the compositional and rheological changes consequent to partial melting are not implemented.

\section{Model predictions}

\section{3.a. Structural configurations}

Figure 3 shows the structural configurations of the crust and lithosphere at different stages of the evolution of the hot model (panels $\mathrm{a}_{\mathrm{i}}$, left side) and the cold model (panels $b_{i}$, right side). The crustal boundaries coincide with the envelope of the crustal type markers. The base of the lithosphere is thermally defined by the $1600 \mathrm{~K}$ isotherm (red dashed line).

During the initial phase of forced extension in both models a progressive thinning of the crust is predicted, mostly concentrated around the position of the future ridge. The crustal thinning occurs very early in the cold model, approximately $1 \mathrm{Ma}$ after the beginning of the extension (Fig. 3 $b_{1}$ ), while more than $10 \mathrm{Ma}$ passes before thinning becomes significant in the hot model (Fig. 3a $\mathrm{a}_{1}$ ). After 15.4 Ma (hot model, Fig. 3a and $4.4 \mathrm{Ma}$ (cold model, Fig. $3 \mathrm{~b}_{2}$ ), the thermal thinning is localized around the position of the future ridge. These times mark the beginning of mantle serpentinization in both models. The long time interval necessary in the hot model for the beginning of deformation localization therefore seems related to the higher thermal state at the base of the continental crust, which results in a different rheological behaviour of the shallow lithospheric mantle as discussed at the end of Section 2.c.

The progression of forced extension leads to the occurrence of the crustal break-up which occurs at 31.4 Ma in the hot model, approximately $16 \mathrm{Ma}$ after the beginning of the mantle serpentinization (Fig. $3 a_{3}$ ), and at 7.4 Ma in the cold model, only $3 \mathrm{Ma}$ after the beginning of the mantle serpentinization (Fig. $3 b_{3}$ ). The occurrence of crustal break-up is followed by the exhumation of the serpentinized mantle replacing the thinned continental crust at the floor of the basin. In the hot model, the mantle serpentinization progresses even at the base of the continental crust for more than $250 \mathrm{~km}$ away from the ridge. In contrast, in the cold model deep-seated serpentinization does not occur. This is a consequence of the thinner continental crust characterizing the hot model, allowing the occurrence of mantle at shallower depths with respect to the cold model in which continental lithospheric mantle resides at greater depths and $P-T$ conditions are inappropriate for serpentinization.

The pressure and temperature conditions at the base of the lithosphere in the hot model are favourable for partial melting for a relatively short time after the crustal break-up (approximately $5 \mathrm{Ma}$, Fig. $3 \mathrm{a}_{4}$ ). In contrast, in the cold model favourable pressure and temperature conditions are reached after a relatively long time from the break-up (approximately $15 \mathrm{Ma}$, Fig. $3 \mathrm{~b}_{4}$ ). Although there are different time intervals between the different stages (thinning, serpentinization and partial melting) in the hot and cold models, once crustal thinning starts comparable time spans pass before the beginning of the mantle partial melting in both models (approximately $21 \mathrm{Ma}$ and $18 \mathrm{Ma}$ for the hot and the cold models, respectively; Fig. 4).

At the beginning of the extension, the low strength of the lithosphere in the hot model reduces the efficiency of stress transmission up to shallow depths, making the localization of crustal thinning slower than that for the stronger lithosphere of the cold model. Once the crustal thinning is localized, the successive evolution is dominated by local thermal gradients because this stage is comparable in the two models. After their formation, the mantle, which is depleted by partial melting (dark yellow markers for the partial melting area and light yellow markers for the depleted mantle in Fig. $3 \mathrm{a}_{5}$, $b_{5}$ ), moves laterally below the expanding oceanic crust, forming the oceanic lithosphere (dark green markers in Fig. $3 a_{5}, b_{5}$ ).

Here we assume that the oceanic lithosphere forms after the beginning of the mantle partial melting, when gabbros, basalts and part of serpentinized mantle contribute to the formation of the oceanic crust. Based on the structural configuration of the system at the time of partial melting (Fig. $3 a_{4}, b_{4}$ ) and after a given time span of $10 \mathrm{Ma}$ (Fig. $3 \mathrm{a}_{5}$ and $\mathrm{b}_{5}$ for the hot model and the cold model, respectively), it is possible to 

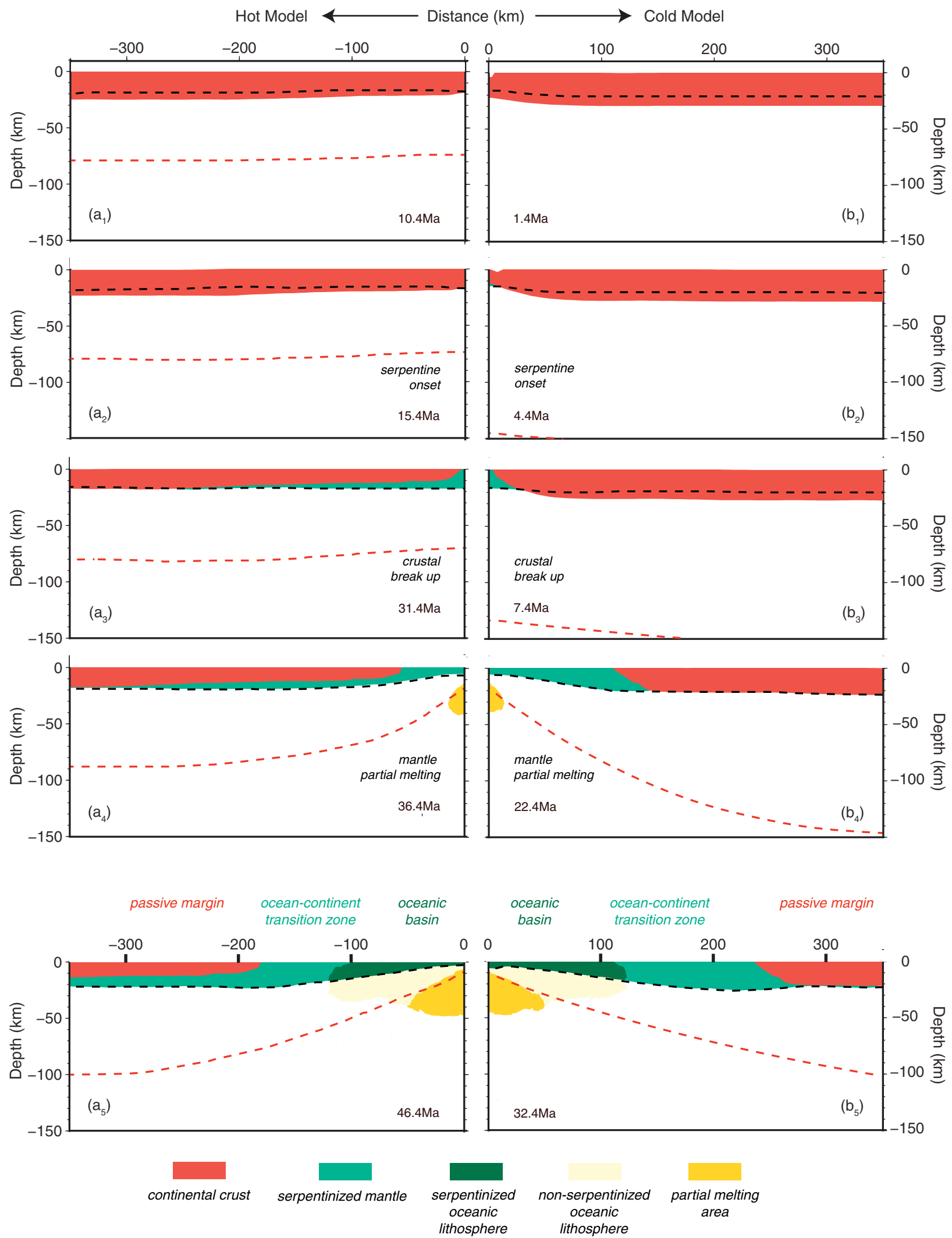

Figure 3. (Colour online) Successive stages of the tectonic evolution predicted by the hot (panels $\mathrm{a}_{\mathrm{i}}$ ) and cold (panels $\mathrm{b}_{\mathrm{i}}$ ) models at different times after the beginning of the forced extension. Black and red dashed lines correspond to $800 \mathrm{~K}$ and $1500 \mathrm{~K}$ isotherms, respectively. Ages refer to the time span from the beginning of the simulations.

estimate the variation in time of the width of the oceanic lithosphere, characterized by a gabbro-basalt-bearing crust, and of the serpentinized mantle denuded before melt generation. In particular, $10 \mathrm{Ma}$ after the onset of the mantle partial melting our results indicate a total basin width ranging over $360-480 \mathrm{~km}$ as a function of the initial thermal state of the lithosphere (hot or cold models, respectively). In both models the oceanic lithosphere extends for approximately $200 \mathrm{~km}$, while the denuded serpentinized mantle covers a width of 


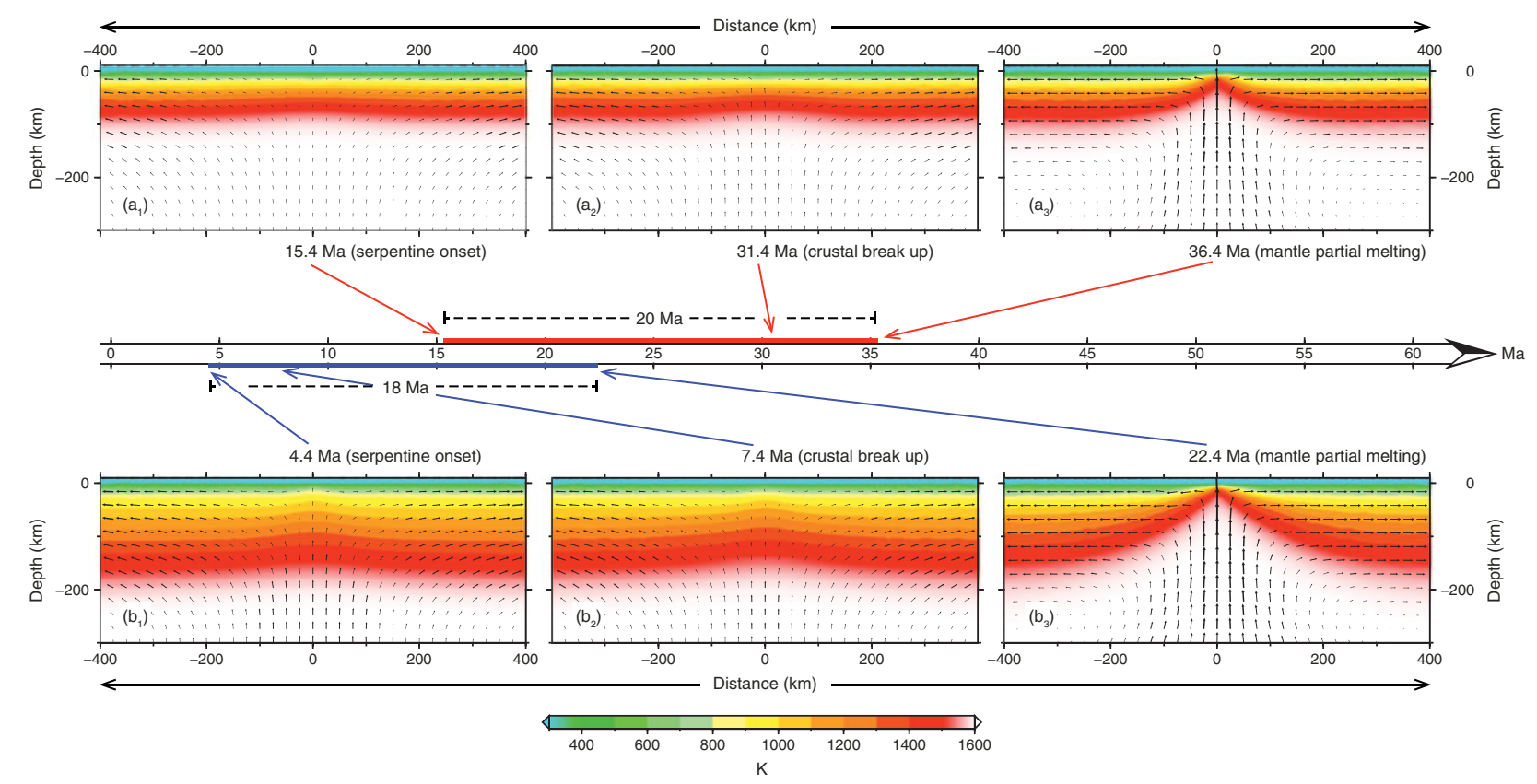

Figure 4. (Colour online) Thermal and velocity fields of the system at different times after the beginning of the forced extension for the hot (panels $\mathrm{a}_{\mathrm{i}}$ ) and cold (panels $\mathrm{b}_{\mathrm{i}}$ ) models. Time scale in the centre of the figure represents the simulation duration for both models, on which the onset of serpentinization, crustal break-up and partial melting are located by arrows (red for the hot and blue for the cold models). Time intervals between serpentinization and partial melting onset are indicated by dashed lines.

approximately $160 \mathrm{~km}$ in the hot model and $280 \mathrm{~km}$ in the cold one.

\section{3.b. Thermo-mechanical evolution}

The structural configuration discussed above can be better understood if the thermo-mechanical evolution of the system is analysed. Figure 4 shows the thermal and velocity fields of the system at different stages of the evolution for the hot and the cold models (Fig. $4 \mathrm{a}_{\mathrm{i}}$ and $b_{i}$, respectively). During the early stages of the evolution of both models, the velocity field is controlled by the far-field traction driving a predominantly horizontal velocity pattern though the lithosphere, being the intensity of the mantle upwelling below the future ridge lower than half the intensity of the far-field for the cold model (panel $b_{1}$ ) and even negligible for the hot model (panel $\mathrm{a}_{1}$ ). Mantle upwelling increases during the evolution (Fig. $4 \mathrm{a}_{2}$ ) and reaches a magnitude comparable to or higher than that of the far-field traction only after the onset of the mantle partial melting (Fig. $4 \mathrm{a}_{3}$ ). For both models, the thermal thinning of the lithosphere localizes at the future ridge position when the mantle serpentinization conditions are matched, at approximately $15 \mathrm{Ma}$ (Fig. $4 \mathrm{a}_{1}$ ) and $4.4 \mathrm{Ma}$ (Fig. 4b $\mathrm{b}_{1}$ ) after the beginning of the forced extension for the hot and cold models, respectively. Thermal thinning achieves its maximum when the pressure and temperature conditions are favourable for the mantle partial melting at $36.4 \mathrm{Ma}$ (Fig. $4 \mathrm{a}_{3}$ ) and $22.4 \mathrm{Ma}$ (Fig. 4b for the hot and cold models, respectively. The thermal thinning in the two models therefore differs only in the initial stages and, once the thermal destabilization starts, the time span needed to reach the maximum thinning is approximately independent of the initial thermal state (thick red and blue lines along the time scale in Fig. 4).

The different behaviours of the cold and hot models during the initial deformation phase and their similar behaviours at longer times can be ascribed to the differences in the lithosphere strength (Fig. 2b). During the initial phase, the lower strength (in terms of the lower effective viscosities of the lithospheric mantle levels) that characterizes the hot model (solid red line in Fig. 2b) may attenuate the transmission of the farfield traction up to the future ridge position, making the lithosphere thinning a very slow process compared with the cold model.

\section{3.c. Velocity configuration}

A more detailed analysis of the surface crustal horizontal velocity (Fig. 5a, b) indicates that both models are characterized by an initial phase during which the mean surface crustal velocity is stationary and increases linearly from the future ridge outwards until it reaches, at the boundary of the model, the velocity value constrained by the far-field traction. This stationary pattern lasts a short time (approximately $5 \mathrm{Ma}$ ) in the cold model (Fig. 5a) and a long time (approximately $30 \mathrm{Ma}$ ) in the hot model (Fig. 5b), ending when the crustal break-up occurs. This initial phase is followed by a transition phase lasting approximately $9 \mathrm{Ma}$ and $3 \mathrm{Ma}$ for the cold and hot models, respectively, during which the far-field and upwelling flow concur to the extension rate and the mean surface 

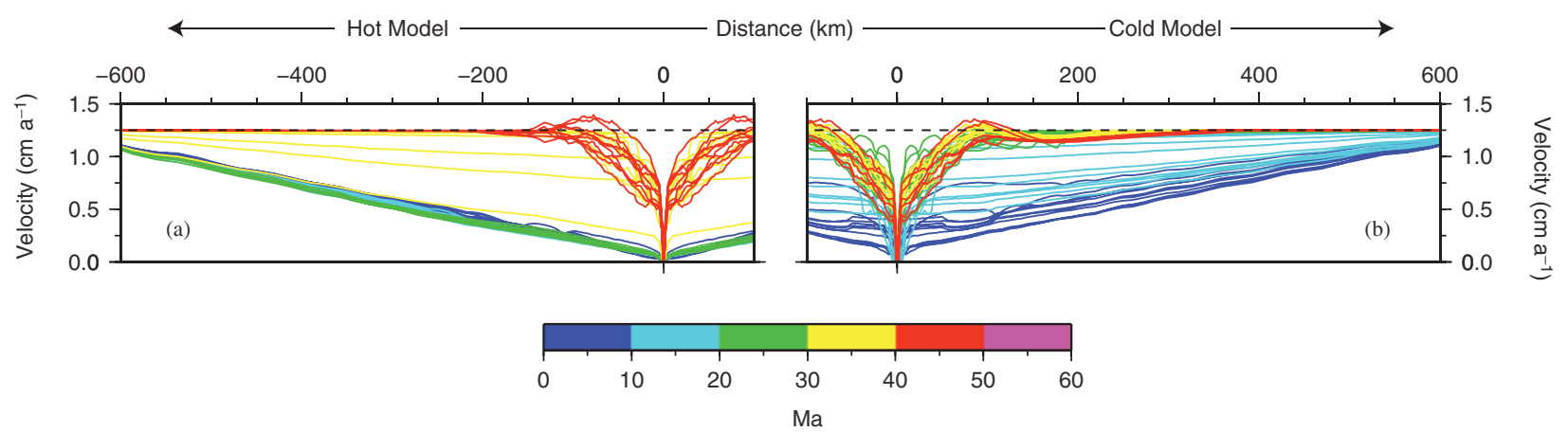

Figure 5. (Colour online) Surface horizontal crustal velocity predicted by the hot (a) and cold (b) models at different times after the beginning of the forced extension.

crustal horizontal velocities progressively increase until they reach the value of the far-field traction velocity. At the advanced stages of evolution the surface crustal horizontal velocity remains almost constant from the ridge to the margins for both models, with the exception of a $150-200 \mathrm{~km}$ wide region around the ridge where the mean surface horizontal velocity of the crust is mainly controlled by the very intense mantle upwelling flow and may overcome the far-field value.

The transmission of the spreading rate towards the periphery of the domain is likely limited by the fixed far-field prescribed velocity at the boundaries of the model producing, at first sight, an artificial local shortening.

Figure 6 shows the maps of the velocity modules through the lithospheric thickness at different time steps of the evolution, for the hot (Fig. $6 \mathrm{a}_{\mathrm{i}}$ ) and cold (Fig. $6 b_{i}$ ) models. Until crustal break-up occurs, the linear increase from the ridge outwards observed in the mean surface crustal velocity (Fig. 5) characterizes the entire lithosphere thickness (Fig. $6 \mathrm{a}_{1}, \mathrm{a}_{2}$ for the hot model and Fig. $6 b_{1}, b_{2}$ for the cold model). A small increase in the velocity is evident within the mantle where a decrease in the viscosity occurs because of the serpentinization. After the crustal break-up, the strongest velocity gradients localize within $200 \mathrm{~km}$ around the ridge and are associated with the increase in the magnitude of the upwelling mantle flow (Fig. 4). In the proximity of the ridge, in both models the extension rate at the base of the crust increases by a factor of 2-4 since the continental break-up to oceanic spreading (compare Fig. $6 a_{2}, b_{2}$ to Fig. $6 a_{3}, b_{3}$ ). Further away, the velocity gradients decrease significantly until they disappear, and the entire continental lithosphere moves as a rigid plate at a rate equal to the traction velocity after the onset of the mantle partial melting. Significant velocity gradients persist within the part of the basin floored by the serpentinized mantle and at the deep lithosphere levels below the ridge, where the intensity of the asthenosphere upwelling overcomes the far-field traction velocity (compare Fig. $4 a_{3}, b_{3}$ to Fig. $6 a_{4}, b_{4}$ ). At the advanced stages of the evolution after the onset of the mantle partial melting, as already observed in Figure 5 for the mean surface horizontal velocity, even the lithosphere velocity overcomes the far-field traction velocity up to a maximum value of $1.4 \mathrm{~cm} \mathrm{a}^{-1}$ compared with the prescribed $1.25 \mathrm{~cm} \mathrm{a}^{-1}$.

\section{Natural data}

The natural data, compared with the model predictions in the following discussion, are derived from continental and oceanic rocks from the Alps and Apennines and include:

1. lithotypes: useful for inferring the continental, oceanic, crustal or mantellic provenance of each considered rock type as well as the raw characterization of the associable lithostratigraphic setting;

2. $P-T$ conditions: useful for individuating potential provenance regions along the model-predicted lithospheric cross-sections based on the occurrence of compatible thermal states; and

3. geochronological data: useful for checking the time correspondences of the mineral assemblage developments with the predicted sequence of thermal states from rifting to oceanic spreading.

The kinematics of the natural structures supported by mineral assemblages, which are used to infer the considered $P-T$ estimates, are also reported as a supplementary check for compatibility with the investigated lithospheric extensional regime.

The rock types, mineral assemblages and climax metamorphic conditions are described in Tables 2-5. In the pre-Mesozoic continental lithosphere of the Alps and Apennines, the metamorphic $T_{\max }$ imprints and igneous activity compatible with the high thermal regime induced by mantle upwelling during lithospheric thinning have Permian-Triassic ages. These estimated conditions have therefore been selected for comparison with model predictions. On the contrary, the age data from the oceanic lithosphere selected for comparison with model predictions are concentrated around Middle Jurassic ages and are referred to gabbro emplacement and metamorphic re-equilibration at the ocean floor.

A synthetic geological outline is given to help readers who are not familiar with the Alps and Apennines to 


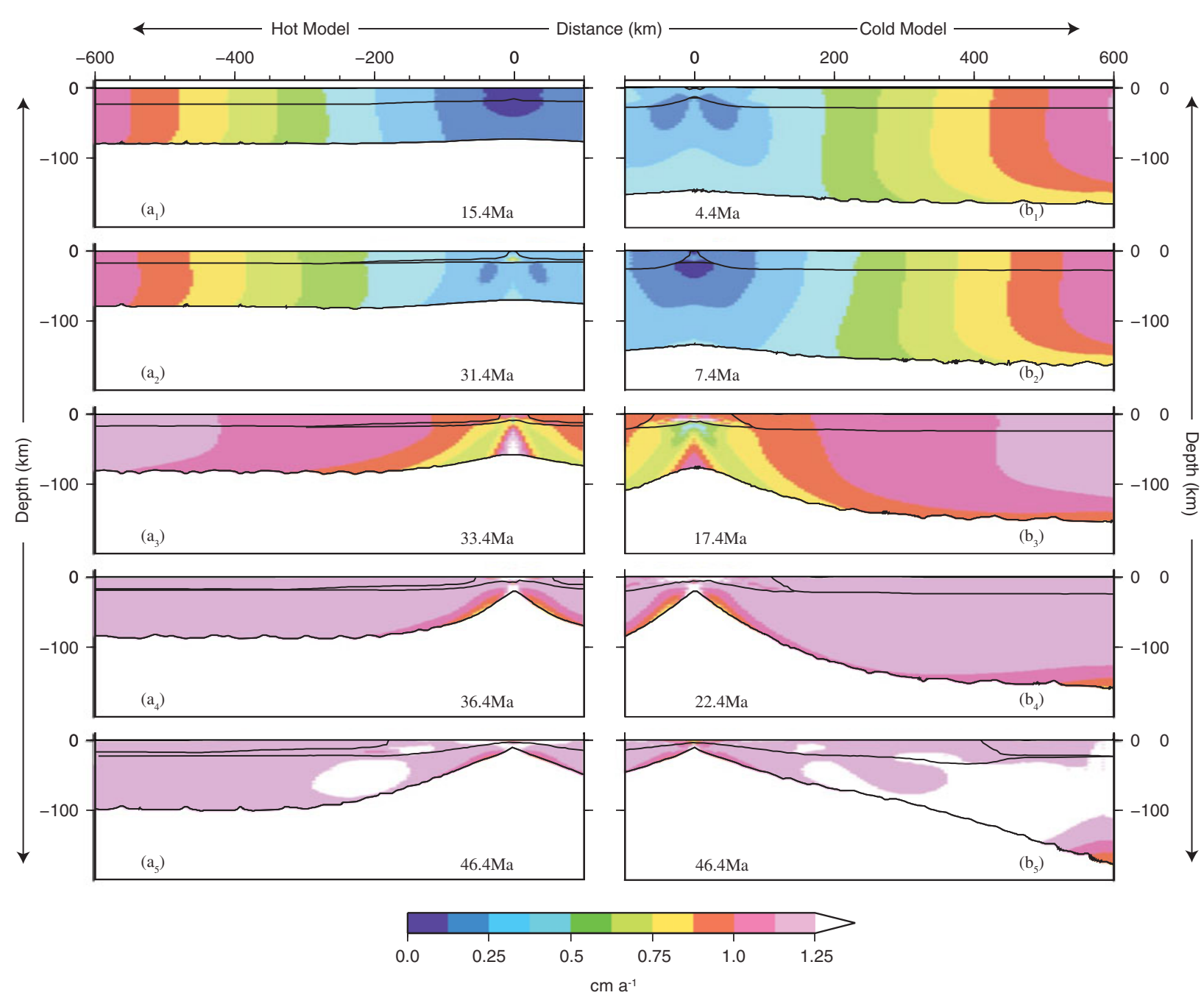

Figure 6. (Colour online) Maps of the velocity modules throughout the lithosphere at different times after the beginning of the forced extension for the hot (panels $\mathrm{a}_{\mathrm{i}}$ ) and cold (panels $\mathrm{b}_{\mathrm{i}}$ ) models.

place the samples that have provided the natural data in an adequate tectonic frame.

\section{4.a. Geological outline}

The Alps and Apennines developed during the closure of the Mesozoic Tethys along two opposite subduction zones during different time intervals, during Cretaceous-Oligocene time for the Alpine system and from Eocene time to the present day for the Apennines system (e.g. Dal Piaz, 2010; Handy et al. 2010; Carminati \& Doglioni, 2012). Different erosion percentages and exhumation efficiencies (Carminati \& Doglioni, 2012) allowed the exposure of deep structural levels in the axial zone of the Alpine nappe; however, these levels are still buried in the Apennines. In contrast to the Apennines, denudation of the axial part of the belt makes the Alps an important site where it is possible to explore continuous sections of deep and intermediate Alpine and pre-Alpine continental crust and to investigate the metamorphic and igneous effects of the lithospheric-thinning-induced high thermal regime preceding Alpine convergence.
The Alps spread out from the Gulf of Genova to the Vienna Basin; the chain was truncated during the Neogenic opening of the Ligurian-Provençal-Algerian and Tyrrhenian basins (e.g. Bozzo et al. 1992; Séranne, 1999; Federico et al. 2009; Turco et al. 2012). Alpine subduction-collision is responsible for the distribution of the continental pre-Alpine and Mesozoic oceanic rocks in four tectonic domains, individuated and explored at the lithospheric scale after the seismic integrated geophysical prospection projects of the whole belt (e.g. Polino, Dal Piaz \& Gosso, 1990; Schmid et al. 2004; Cassinis, 2006). From the internal to the external part of the belt, they are the Southalpine, Austroalpine, Penninic and Helvetic domains (Fig. 1).

The Southalpine domain consists of a south-verging thrust system that has been active since Cretaceous time, involving Palaeozoic continental basement and Permian-Cenozoic cover units, both only locally affected by very-low-grade Alpine metamorphism. The Austroalpine and Penninic domains have been deeply involved in the Alpine subduction and collision system, as accounted for by the high-pressure metamorphic imprints associated with the dominant Alpine 
Table 2. Permian-Triassic metamorphic rocks from the continental crust of the Alps. Labels listed in the code column are reported in Figures 1 and 9-11 to indicate rock positions and duration of thermal fitting

\begin{tabular}{|c|c|c|c|c|c|c|c|c|}
\hline Tectonic unit, location & Lithology & Assemblage & $T\left({ }^{\circ} \mathrm{C}\right)$ & $P(\mathrm{GPa})$ & Age (Ma) & Method & References & Code \\
\hline $\begin{array}{l}\text { Penninic } \\
\text { Briancon basement, } \\
\text { Ruitor }\end{array}$ & Metapelites & Ab-bearing metapelites & $450-550$ & $0.1-0.3$ & Permian (295-245) & $\mathrm{Rb}-\mathrm{Sr} \mathrm{K}-\mathrm{Ar}$ & $\begin{array}{l}\text { Bocquet et al. 1974; Desmons, } \\
1992\end{array}$ & Pp1 \\
\hline Dora Maira & Metapelites & Grt, Sil, Bt, Pl, Qtz & $650-750$ & $0.4-0.7$ & Permian? (295-245) & & Bouffette et al. 1993 & $\mathrm{Pp} 3$ \\
\hline Gruf & Acid granulites & $\begin{array}{l}\text { Qtz, Pl, Ksp, Bt, Opx, } \\
\text { Grt, Amph, Zrc, Rt, } \\
\text { Ilm, Op, } \pm \text { Spl }\end{array}$ & $920-940$ & $0.85-0.95$ & Permian (290-260) & $\mathrm{U}-\mathrm{Pb}$ & Galli et al. 2012 & Pp4 \\
\hline \multicolumn{9}{|l|}{ Austroalpine } \\
\hline $\begin{array}{l}\text { Sesia Lanzo Zone } \\
\text { lower element }\end{array}$ & Basic granulites & $\begin{array}{l}\text { Opx, Pl, Grt, Qtz, } \\
\text { Amph }\end{array}$ & $650-800$ & $0.7-0.9$ & Permian? (295-245) & & Lardeaux \& Spalla, 1991 & Ap1a \\
\hline $\begin{array}{l}\text { Sesia Lanzo Zone } \\
\text { lower element }\end{array}$ & Acidic granulites & Sill, Bt, Cd, Pl, Qtz & $650-750$ & $0.6-0.8$ & Permian? (295-245) & & Lardeaux \& Spalla, 1991 & Ap1b \\
\hline $\begin{array}{l}\text { Sesia Lanzo Zone } \\
\text { upper element }\end{array}$ & $\begin{array}{l}\text { Basic and acidic } \\
\text { granulites }\end{array}$ & $\begin{array}{l}\text { Opx, Pl, Grt, Qtz, } \\
\text { Amph Sill, Bt, Pl, } \\
\text { Qtz }\end{array}$ & $650-750$ & $0.6-0.7$ & Permian? (295-245) & & $\begin{array}{l}\text { Lardeaux, 1981; Vuichard, } \\
\text { 1987; Biagini et al. } 1995\end{array}$ & Ap2 \\
\hline Mt Emilius Klippe & Metabasics & Amph, Pl, Qtz & $550-650$ & $0.3-0.45$ & Permian? (280?) & & $\begin{array}{l}\text { Dal Piaz, Lombardo \& Gosso, } \\
1983\end{array}$ & Ap3 \\
\hline Mont Mary Nappe & Metapelites & Grt, Sil, Bt, Pl, Ms & $510-580$ & $0.25-0.45$ & Permian? (295-245) & & Pennacchioni \& Cesare, 1997 & Ap4 \\
\hline $\begin{array}{l}\text { Dent Blanche Nappe } \\
\text { (Valpelline) }\end{array}$ & $\begin{array}{l}\text { Basic and acidic } \\
\text { granulites }\end{array}$ & $\begin{array}{l}\text { Opx, Pl, Grt, Qtz, } \\
\text { Amph Sill, Bt, Cd, } \\
\text { Pl, Qtz }\end{array}$ & $750-800$ & $0.5-0.7$ & Jurassic? $(\geq 180)$ & $\mathrm{K}-\mathrm{Ar}$ & $\begin{array}{l}\text { Nicot, 1977; Hunziker, Desmon } \\
\text { \& Hurford, 1992; Gardien, } \\
\text { Reusser \& Marquer, } 1994\end{array}$ & Ap $5 b$ \\
\hline Dent Blanche & Metapelites & Qtz, Kfs, Pl, Grt \pm Crd & $814 \pm 40$ & $0.6-0.8$ & Permian (268-272) & $\mathrm{U}-\mathrm{Pb}$ & Manzotti \& Zucali, 2013 & Ap7 \\
\hline Dent Blanche & Metapelites & $\mathrm{Bt}, \mathrm{Kfs}, \mathrm{Qz}, \mathrm{Sil}$, Grt & $750 \pm 50$ & $0.4-0.5$ & Permian (287-291) & $\mathrm{U}-\mathrm{Pb}$ & Manzotti \& Zucali, 2013 & Ap8 \\
\hline Languard-Campo & Granulites & Sil, Opx, Kfs, Bt, Qtz & $570-750$ & $0.4-0.6$ & Permian (288-292) & $\mathrm{SmNd}$ & $\begin{array}{l}\text { Giacomini et al. 1999; Tribuzio, } \\
\text { Thirwall \& Messiga, 1999; } \\
\text { Schuster } \text { et al. 2001 }\end{array}$ & Ap10 \\
\hline Languard-Campo & $\begin{array}{l}\text { Metapelites and } \\
\text { metabasics }\end{array}$ & $\begin{array}{l}\text { Sill, Bt, Grt, Cd, Pl, } \\
\text { Qtz Amph, Grt, Cpx, } \\
\text { Pl, Qtz }\end{array}$ & $650-750$ & $0.4-0.5$ & Permian (260-280) & $\mathrm{Rb}-\mathrm{Sr}$ & $\begin{array}{l}\text { Spalla, Messiga \& Gosso, 1995; } \\
\quad \text { Zucali, } 2001\end{array}$ & Ap11 \\
\hline Matsch Nappe & Metapelites & $\begin{array}{l}\text { Grt, Sil/And, Bt, Pl, } \\
\text { Qtz } \pm \text { Crd }\end{array}$ & $570-640$ & $0.3-0.55$ & Permian $(290 \pm 17)$ & $\mathrm{Rb}-\mathrm{Sr}$ & Gregnanin, 1980; Haas, 1985 & Ap12 \\
\hline Uttenheim Ahrntal & Metapelites & Grt, Bt, Sil, Pl, Qtz, L & $620-680$ & $0.5-0.7$ & Permo-Trias $(262 \pm 7253 \pm 7)$ & $\mathrm{Rb}-\mathrm{Sr} \mathrm{Sm} / \mathrm{Nd}$ & $\begin{array}{l}\text { Borsi et al. 1968; Stöckhert, } \\
\text { 1987; Schuster } \text { et al. } 2001\end{array}$ & Ap13 \\
\hline $\begin{array}{l}\text { Strieden } \\
\quad \text { Kreuzeck-gruppe }\end{array}$ & Metapelites & Sil, Bt, Pl, Qtz, L & $600-750$ & $0.3-0.5$ & Permo-Trias $(261 \pm 3229 \pm 3)$ & $\mathrm{Sm} / \mathrm{Nd} \mathrm{K}-\mathrm{Ar}$ & $\begin{array}{l}\text { Hoke, 1990; Schuster et al. } \\
\quad 2001\end{array}$ & Ap14 \\
\hline Woelz Complex & Gneiss & $\begin{array}{l}\text { Grt, Chl, Ms/Pg, Ab, } \\
\text { Qtz, } \pm \mathrm{Bt}, \pm \mathrm{Mrg}\end{array}$ & $440-520$ & $0.2-0.4$ & Permo-Trias (220-260) & $\mathrm{Rb}-\mathrm{Sr}$ & Schuster \& Frank, 1999 & Ap15 \\
\hline Woelz Complex & Metapelites & $\begin{array}{l}\text { Grt, Bt, Ms, Ilm/Rt, Pl, } \\
\text { Qtz }\end{array}$ & $515-555$ & $0.35-0.45$ & Permo-Trias (295-245) & & Gaidies et al. 2006 & Ap16 \\
\hline Sopron & Metapelites & Bt, And/Sill, Qtz, Pl & $575-700$ & $0.18-0.38$ & Permian? $(300 \pm 40)$ & Th-U-total Pb & Nagy et al. 2002 & Ap17 \\
\hline Saualpe-Koralpe & $\begin{array}{l}\text { Metapelites and } \\
\text { pegmatites }\end{array}$ & $\begin{array}{l}\text { Grt-bearing } \\
\text { micaschists }\end{array}$ & $550-560$ & $0.52-0.71$ & Permo-Trias (240-290) & $\mathrm{Rb}-\mathrm{Sr} \mathrm{U}-\mathrm{Pb} \mathrm{Sm} / \mathrm{Nd}$ & Thöni \& Miller, 2000 & Ap19a \\
\hline Saualpe-Koralpe & Metapelites & $\begin{array}{l}\text { Grt, Qtz, Pl, Bt, Ms, } \pm \\
\text { Sil }\end{array}$ & $575-620$ & $0.3-0.5$ & Triassic $(249 \pm 3)$ & $\mathrm{Sm} / \mathrm{Nd}$ & Habler \& Thöni, 2001 & Ap19b \\
\hline Saualpe-Koralpe & Metapelites & $\begin{array}{l}\text { Grt, Qtz, Pl, Bt, Ms, } \pm \\
\text { Sil }\end{array}$ & $650-670$ & $0.4-0.9$ & Permo-Trias (290-245) & & Tenczer, Powell \& Stuwe, 2006 & Ap19c \\
\hline
\end{tabular}


Table 2. Continued

\begin{tabular}{|c|c|c|c|c|c|c|c|c|}
\hline Tectonic unit, location & Lithology & Assemblage & $T\left({ }^{\circ} \mathrm{C}\right)$ & $P(\mathrm{GPa})$ & Age (Ma) & Method & References & Code \\
\hline $\begin{array}{l}\text { Saualpe-Koralpe } \\
\text { (Plankogel) }\end{array}$ & Micaschists & $\begin{array}{l}\text { Ky, St, Grt, Qtz, Wm, } \\
\text { Bi, Chl, Ru, Ilm }\end{array}$ & $550 \pm 50$ & $0.36-0.56$ & Permian $(269.6 \pm 6.2)$ & $\mathrm{Sm} / \mathrm{Nd}$ & Thöni \& Miller, 2009 & Ap21 \\
\hline $\begin{array}{l}\text { Southalpine } \\
\text { Eisacktal }\end{array}$ & $\begin{array}{l}\text { Metapelites- } \\
\text { contact } \\
\text { meta-morphism }\end{array}$ & Crd, Sil, Bt & $500-630$ & $0.1-0.26$ & Permian (282) & & $\begin{array}{l}\text { Visonà, 1995; Benciolini et al. } \\
\quad 2006\end{array}$ & Sp1 \\
\hline Dervio-Olgiasca Zone & $\begin{array}{l}\text { Metapelites } \\
\text { metabasics }\end{array}$ & $\begin{array}{l}\mathrm{Bt}, \mathrm{Sil}, \mathrm{Pl}, \mathrm{Qtz}, \pm \mathrm{Grt} \\
\quad \pm \mathrm{Kfs} \text { Amph, } \mathrm{Pl}, \mathrm{Qtz} \\
\quad \pm \mathrm{Cpx}, \pm \mathrm{Bt}\end{array}$ & $650-750$ & $0.4-0.5$ & Triassic (224-227) & $\mathrm{Rb}-\mathrm{Sr}$ & $\begin{array}{l}\text { Diella, Spalla \& Tunesi, 1992; } \\
\text { Bertotti et al. 1993; Sanders, } \\
\text { Bertotti \& Tommasini, 1996; } \\
\text { di Paola \& Spalla, 2000 }\end{array}$ & Sp3 \\
\hline Strona-Ceneri Zone & Metapelites & Sil, Ad, Crd & & & Permo-Trias (288 \pm 99$)$ & $\mathrm{Rb}-\mathrm{Sr}$ best-fit & $\begin{array}{l}\text { Boriani \& Burlini, 1995; } \\
\quad \text { Pinarelli \& Boriani, } 2007\end{array}$ & Sp4 \\
\hline Ivrea Zone & Metapelites & Sill, Bt, Grt, Cd, Pl, Qtz & $680-780$ & $0.45-0.65$ & Permian (250-290) & $\mathrm{Rb}-\mathrm{Sr} \mathrm{U}-\mathrm{Pb}$ & $\begin{array}{l}\text { Hunziker \& Zingg, 1980; } \\
\text { Brodie et al. 1989; Quick } \\
\text { et al. 1992; Vavra et al. 1996; } \\
\text { Colombo \& Tunesi, } 1999\end{array}$ & Sp5a \\
\hline Ivrea Zone & Metabasics & $\begin{array}{l}\text { Grt, Opx, Amph, Pl, } \\
\text { Qtz }\end{array}$ & $750-950$ & $0.8-0.9$ & Permian (273-296) & & $\begin{array}{l}\text { Henk et al. 1997; Vavra et al. } \\
\text { 1996; Colombo \& Tunesi, } \\
\text { 1999 }\end{array}$ & $\mathrm{Sp} 5 \mathrm{~b}$ \\
\hline Ivrea Zone & Granulites & Grt, Sil, Pl, Qtz, Rt & $760-810$ & $0.45-1.05$ & Permian $(288 \pm 4)$ & $\mathrm{U}-\mathrm{Pb}$ & Smye \& Stockli, 2014 & Sp5 \\
\hline Ivrea Zone & Metapelites & $\begin{array}{l}\text { Gt, Sil, Rt, Qtz, Fsp, } \\
\text { Pl, Bt }\end{array}$ & $900-1000$ & $0.75-1.25$ & Permian? $(316 \pm 3)$ & $\mathrm{U}-\mathrm{Pb}$ & $\begin{array}{l}\text { Ewing, Hermann \& Rubatto, } \\
2013\end{array}$ & Sp5 \\
\hline Ivrea Zone & Metapelites & $\begin{array}{l}\text { Gt, Sil, Rt, Qtz, Fsp, } \\
\text { Pl, Bt }\end{array}$ & $700-800$ 810-870 & $0.35-1.15$ & Permian $(276 \pm 4258 \pm 3)$ & $\mathrm{U}-\mathrm{Pb}$ & $\begin{array}{l}\text { Ewing, Hermann \& Rubatto, } \\
2013\end{array}$ & Sp5 \\
\hline Ivrea Zone & Metapelites & $\begin{array}{l}\text { Amph, CPx, Pl, } \pm \mathrm{Qtz} \\
\quad \pm \mathrm{Bt}, \pm \mathrm{Gt}, \pm \text { Sill }\end{array}$ & & & Permian $(284 \pm 14)$ & $\mathrm{U}-\mathrm{Th}-\mathrm{Pb}$ & Langone \& Tiepolo, 2015 & Sp6 \\
\hline Ivrea Zone & Metapelites & $\begin{array}{l}\text { Amph, CPx, Pl, } \pm \mathrm{Qtz} \\
\pm \mathrm{Bt}, \pm \mathrm{Gt}, \pm \text { Sill }\end{array}$ & & & Triassic $(234 \pm 8)$ & $\mathrm{U}-\mathrm{Th}-\mathrm{Pb}$ & Langone \& Tiepolo, 2015 & Sp6 \\
\hline Ivrea Zone & Metapelites & $\begin{array}{l}\text { Amph, CPx, Pl, } \pm \mathrm{Qtz} \\
\quad \pm \mathrm{Bt}, \pm \mathrm{Gt}, \pm \text { Sill }\end{array}$ & & & Jurassic $(154 \pm 12)$ & $\mathrm{U}-\mathrm{Th}-\mathrm{Pb}$ & Langone \& Tiepolo, 2015 & Sp6 \\
\hline
\end{tabular}


Table 3. Permian-Triassic gabbros emplaced in the pre-Alpine continental crust of the Alps and Apennines. Labels listed in the code column are reported in Figures 1 and $9-11$ to indicate rock positions and duration of thermal fitting

\begin{tabular}{|c|c|c|c|c|c|c|c|c|}
\hline Tectonic unit, location & Lithology & Assemblage & $T\left({ }^{\circ} \mathrm{C}\right)$ & $P(\mathrm{GPa})$ & Age (Ma) & Method & References & Code \\
\hline \multicolumn{9}{|l|}{ Austroalpine } \\
\hline Sesia Lanzo, Corio and Monastero & Gabbro-norite & $\begin{array}{l}\text { Cpx, Opx, Pl, Amph, } \\
\text { Ilm, Ap }\end{array}$ & $780-920$ & $0.6-0.9$ & Permian? & Geolog. evidence & Rebay \& Spalla, 2001 & g1 \\
\hline Sesia Lanzo, Sermenza & Gabbro & $\begin{array}{l}\mathrm{Pl}, \mathrm{Amph}, \mathrm{Cpx}, \mathrm{Mt} \text {, } \\
\text { Zo, } \pm \mathrm{Wm}, \mathrm{Chl}\end{array}$ & & & Permian $(288+2 /-4)$ & $\mathrm{U}-\mathrm{Pb}$ & Bussy et al. 1998 & g2 \\
\hline Dent Blanche, Matterhorn Collon & Gabbro & $\begin{array}{l}\text { Ol, Opx, Sp, Cpx, Pl, } \\
\text { Amph, Phl }\end{array}$ & & & Triassic $(250 \pm 5)$ & $\mathrm{K}-\mathrm{Ar} \mathrm{Rb}-\mathrm{Sr}$ & $\begin{array}{l}\text { Dal Piaz, De Vecchi \& } \\
\text { Hunziker, } 1977\end{array}$ & g3 \\
\hline Dent Blanche, Matterhorn Collon & Gabbro & $\begin{array}{l}\text { Ol, Cpx, Pl, Opx, } \\
\text { Amph, Ilm, Mt }\end{array}$ & $1070-1120$ & $0.5-0.7$ & Permian $(284 \pm$ & $\mathrm{U}-\mathrm{Pb}$ & $\begin{array}{l}\text { Monjoie, 2004; Monjoie et al. } \\
2005\end{array}$ & g3 \\
\hline $\begin{array}{l}\text { Dent Blanche, Mont Collon Dents } \\
\text { de Bertol }\end{array}$ & $\begin{array}{l}\text { Mafic dykes } \\
\text { (alkaline } \\
\text { lamprophyres) }\end{array}$ & Amph, Cpx, Pl, Ilm, & & & Permian (c. 260) & $\mathrm{Ar}-\mathrm{Ar}$ & $\begin{array}{l}\text { Monjoie, 2004; Monjoie et al. } \\
2005\end{array}$ & g3 \\
\hline Fedoz, Braccia & Gabbro & $\begin{array}{l}\text { Ol, Cpx, Pl, Opx, } \\
\text { Amph, Ilm }\end{array}$ & $1150-1250$ & $1.0-1.2$ & Permian (266-276) & $\mathrm{U}-\mathrm{Pb}$ & $\begin{array}{l}\text { Muentener, Hermann \& } \\
\text { Trommsdorf, } 2000\end{array}$ & $\mathrm{~g} 4$ \\
\hline Fedoz, Braccia & Gabbro & $\begin{array}{l}\text { Ol, Cpx, Pl, Opx, } \\
\text { Amph, Ilm, Mt }\end{array}$ & & & Permian $(281 \pm 19,281 \pm 2)$ & $\mathrm{U}-\mathrm{Pb}$ & $\begin{array}{l}\text { Hansmann, Muntener \& } \\
\text { Hermann, 2001; Hermann \& } \\
\text { Rubatto, } 2003\end{array}$ & $\mathrm{~g} 4$ \\
\hline Sondalo & Gabbro & $\mathrm{Pl}, \mathrm{Ol}, \mathrm{Cpx}$ & $800-900$ & $0.4-0.6$ & Permian $(267 \pm 13)$ & $\mathrm{Rb}-\mathrm{Sr}$ & $\begin{array}{l}\text { Boriani, Colombo \& Macera, } \\
\text { 1985; Tribuzio, Thirwall \& } \\
\text { Messiga, } 1999\end{array}$ & g5 \\
\hline Sondalo & Troctolite & $\begin{array}{l}\mathrm{Pl}, \mathrm{Cpx}, \mathrm{Amph}, \mathrm{Bt}, \\
\mathrm{Opx}\end{array}$ & & $0.3-0.7$ & Permian $(266 \pm 10,300 \pm 12)$ & $\mathrm{Rb}-\mathrm{Sr} \mathrm{Sm}-\mathrm{Nd}$ & $\begin{array}{l}\text { Tribuzio, Thirwall \& Messiga, } \\
1999\end{array}$ & g5 \\
\hline Sondalo & Norite & $\begin{array}{l}\text { Pl, Opx, Cpx, Amph, } \\
\text { Ilm, Bt }\end{array}$ & & & Permian $(269 \pm 16,280 \pm 10)$ & $\mathrm{Rb}-\mathrm{Sr} \mathrm{Sm}-\mathrm{Nd}$ & $\begin{array}{l}\text { Tribuzio, Thirwall \& Messiga, } \\
1999\end{array}$ & g5 \\
\hline Baerofen & Gabbro & $\mathrm{Pl}, \mathrm{Cpx}, \mathrm{Ol}, \mathrm{Opx}$ & & & Permian $(275 \pm 18)$ & $\mathrm{Sm}-\mathrm{Nd}$ & Thöni \& Jagoutz, 1992 & g6 \\
\hline Baerofen & Gabbro & $\mathrm{Pl}, \mathrm{Cpx}, \mathrm{Ol}, \mathrm{Opx}$ & & & Permo-Trias $(261 \pm 10)$ & $\mathrm{Sm}-\mathrm{Nd}$ & Thöni \& Jagoutz, 1992 & g6 \\
\hline Baerofen and Gressenberg & Eclogitic gabbro & $\mathrm{Pl}, \mathrm{Cpx}, \mathrm{Ol}$ & 1100 & 0.25 & Permo-Trias $(247 \pm 16,255 \pm 9)$ & $\mathrm{Sm}-\mathrm{Nd}$ & Miller \& Thöni, 1997 & g6 \\
\hline \multicolumn{9}{|l|}{ Southalpine } \\
\hline Bressanone, Chiusa dioritic belt & Gabbro-norite & $\begin{array}{l}\text { Cpx, Pl, Amph, Opx, } \\
\text { Bt, Mt, Ilm, Ol, Qtz }\end{array}$ & $900-1200$ & $0.1-0.3$ & Permian $(276 \pm 4)$ & $\mathrm{Rb}-\mathrm{Sr}$ & $\begin{array}{l}\text { Del Moro \& Visonà, 1982; } \\
\text { Visonà, } 1995\end{array}$ & g7 \\
\hline Monzoni & Gabbro & $\mathrm{Pl}, \mathrm{Cpx}, \mathrm{Bt}$, kfsp, Ol & $960-990$ & 0.1 & Triassic (234-225) & $\mathrm{Rb}-\mathrm{Sr}$ & $\begin{array}{l}\text { Borsi et al. 1968; Spicker and } \\
\text { Huckenholz, 1986; Povoden, } \\
\text { Horacek \& Abart, } 2002\end{array}$ & g8 \\
\hline Predazzo & Gabbro, diorite & $\begin{array}{l}\text { Cpx, Opx, Amph, Pl, } \\
\text { Bt, Mt, Ilm, Sph, Ap, } \\
\text { Ol }\end{array}$ & & & Triassic (238-232) & $\mathrm{U}-\mathrm{Pb} \mathrm{Ar}-\mathrm{Ar}$ & $\begin{array}{l}\text { Mundil, Brack \& Laurenzi, } \\
\text { 1996; Visonà, 1997; Ferry } \\
\text { et al. } 2002\end{array}$ & g8 \\
\hline Val Biandino & Gabbro, diorite & $\begin{array}{l}\mathrm{Pl}, \mathrm{Bt}, \mathrm{Amph}, \mathrm{Qtz} \\
\text { Zirc, Op, Apa }\end{array}$ & $700-825$ & $0.25-0.4$ & Permian $(279 \pm 5)$ & $\mathrm{Rb}-\mathrm{Sr}$ & $\begin{array}{l}\text { Thöni et al. 1992; De Capitani, } \\
\text { Carnevale \& Fumagalli, } 2007\end{array}$ & g9 \\
\hline $\begin{array}{l}\text { Ivrea, Upper Mafic Complex, Val } \\
\text { Mastallone }\end{array}$ & Diorite & $\begin{array}{l}\text { Cpx, Opx, Pl, Amph, } \\
\text { Bt, Grt, Qtz, K-Fld, }\end{array}$ & & & Permian $(285+7 /-5)$ & $\mathrm{U}-\mathrm{Pb}$ & Pin, 1986 & g10 \\
\hline $\begin{array}{l}\text { Ivrea, Upper Mafic Complex, Val } \\
\text { Sesia, Val Strona }\end{array}$ & Diorite & Qtz, Fld, Grt, Bt, Opx & & & Permian $(274 \pm 17)$ & $\mathrm{Rb}-\mathrm{Sr}$ & Buergi \& Kloetzli, 1990 & g10 \\
\hline Ivrea, Upper Mafic Complex & Gabbro, diorite & $\begin{array}{l}\text { Amph, Ol, Opx, Cpx, } \\
\text { Phl, Pl }\end{array}$ & & & Permian $(287 \pm 3,292 \pm 4)$ & $\mathrm{U}-\mathrm{Pb}$ & Garuti et al. 2001 & g10 \\
\hline $\begin{array}{l}\text { Ivrea, Upper Mafic Complex, Val } \\
\text { Mastallone and Val Sesia }\end{array}$ & Diorite and gabbro & $\begin{array}{l}\text { Pl, Opx, Cpx, Bt, } \\
\text { Amph }\end{array}$ & & & Permian $(288 \pm 3)$ & $\mathrm{U}-\mathrm{Pb}$ & Peressini et al. 2007 & $\mathrm{~g} 10$ \\
\hline
\end{tabular}


Table 3. Continued

\begin{tabular}{|c|c|c|c|c|c|c|c|c|}
\hline Tectonic unit, location & Lithology & Assemblage & $T\left({ }^{\circ} \mathrm{C}\right)$ & $P(\mathrm{GPa})$ & Age (Ma) & Method & References & Code \\
\hline $\begin{array}{l}\text { Ivrea, Upper Mafic Complex, } \\
\text { Valbella }\end{array}$ & Gabbro & Pl, Cpx, Opx, Amph & & & Permo-Trias $(271 \pm 22)$ & $\mathrm{Sm}-\mathrm{Nd}$ & Voshage et al. 1987 & g10 \\
\hline $\begin{array}{l}\text { Ivrea, Upper Mafic Complex, } \\
\text { Sassiglioni }\end{array}$ & Gabbro & Pl, Cpx Opx, Amph & $1000-1200$ & $0.55-0.75$ & Triassic $(248 \pm 8)$ & $\mathrm{Sm}-\mathrm{Nd}$ & Voshage et al. 1987 & g10 \\
\hline $\begin{array}{l}\text { Ivrea, Lower Mafic Complex, Val } \\
\text { Sesia }\end{array}$ & Gabbro & $\mathrm{Pl}$, Amph, Cpx, Opx & $>750$ & 0.8 & Permian $(274 \pm 11)$ & $\mathrm{Sm}-\mathrm{Nd}$ & Mayer, Mezger \& Sinigoi, 2000 & g10 \\
\hline $\begin{array}{l}\text { Ivrea, Upper Mafic Complex, Val } \\
\text { Sessera }\end{array}$ & Gabbro & $\begin{array}{l}\text { Pl, Amph, Cpx Opx, } \\
\text { Bt, Ap, Zrc, Spl, Ilm }\end{array}$ & & & Permo-Trias $(267 \pm 21)$ & $\mathrm{Sm}-\mathrm{Nd}$ & Mayer, Mezger \& Sinigoi, 2000 & g10 \\
\hline Ivrea, Upper Mafic Complex & Metabasics & $\begin{array}{l}\text { Qtz, Pl, Grt, Bt, Sil, } \\
\text { Opx }\end{array}$ & & & Permian $(293 \pm 12)$ & $\mathrm{U}-\mathrm{Pb}$ & Vavra, Schmid \& Gebauer, 1999 & g10 \\
\hline Ivrea, Verbano & Gabbro & & & & Permian $(287 \pm 2285 \pm 4)$ & $\mathrm{U}-\mathrm{Pb}$ & Quick et al. 2002 & $\mathrm{~g} 10$ \\
\hline Ivrea, Finero mafic ultramafic body & Gabbro & Grt, Cpx, Pl, Amph & & & Triassic $(231 \pm 21223 \pm 10)$ & $\mathrm{Sm}-\mathrm{Nd}$ & Lu et al. 1997 & $\mathrm{~g} 11$ \\
\hline Ivrea, Finero mafic ultramafic body & Gabbro & Grt, Cpx, Pl, Amph & & & Triassic $(215 \pm 15)$ & $\mathrm{Sm}-\mathrm{Nd}$ & Lu et al. 1997 & $\mathrm{~g} 11$ \\
\hline Ivrea, Finero mafic ultramafic body & Gabbro & Grt, Cpx, Pl, Amp & $850-1100$ & $0.9-1$ & Triassic $(231 \pm 21,223 \pm 10)$ & $\mathrm{Sm}-\mathrm{Nd}$ & Lu et al. 1997 & g11 \\
\hline Ivrea, Finero mafic ultramafic body & Gabbro & Grt, Cpx, Pl, Amp & & & Triassic $(215 \pm 15)$ & $\mathrm{Sm}-\mathrm{Nd}$ & Lu et al. 1997 & $\mathrm{~g} 11$ \\
\hline $\begin{array}{l}\text { Ponte Gardena, Waidbruck (Isarco, } \\
\text { Eisack valley) }\end{array}$ & Basaltic andesite & $\mathrm{Pl}, \mathrm{Px}, \mathrm{Bt}$ & & & Permian $(290.7 \pm 3)$ & $\mathrm{U}-\mathrm{Pb}$ & Visonà et al. 2007 & $\mathrm{~g} 12$ \\
\hline $\begin{array}{l}\text { M dei Ginepri (Eores, Aferer } \\
\text { valley) }\end{array}$ & Andesitic necks & Pl, Px, Bt, Mag, Grt & & & Permian $(279.9 \pm 3.3)$ & $\mathrm{U}-\mathrm{Pb}$ & Visonà et al. 2007 & g12 \\
\hline Col Quaternà (western Comelico) & Andesitic necks & Pl, Px, Amph & & & Permian $(278.6 \pm 3.1)$ & $\mathrm{U}-\mathrm{Pb}$ & Visonà et al. 2007 & g12 \\
\hline $\begin{array}{l}\text { Apennine } \\
\text { External Liguride Northern } \\
\text { Apennine }\end{array}$ & Granulitic gabbro & $\begin{array}{l}\text { Ol, Pl, Cpx, Opx, Par, } \\
\text { Sp }\end{array}$ & $810-920$ & $0.7-0.8$ & Permian $(291 \pm 9)$ & $\mathrm{Sm}-\mathrm{Nd}$ & $\begin{array}{l}\text { Marroni and Tribuzio, 1996; } \\
\text { Marroni et al. } 1998\end{array}$ & APN2a \\
\hline
\end{tabular}




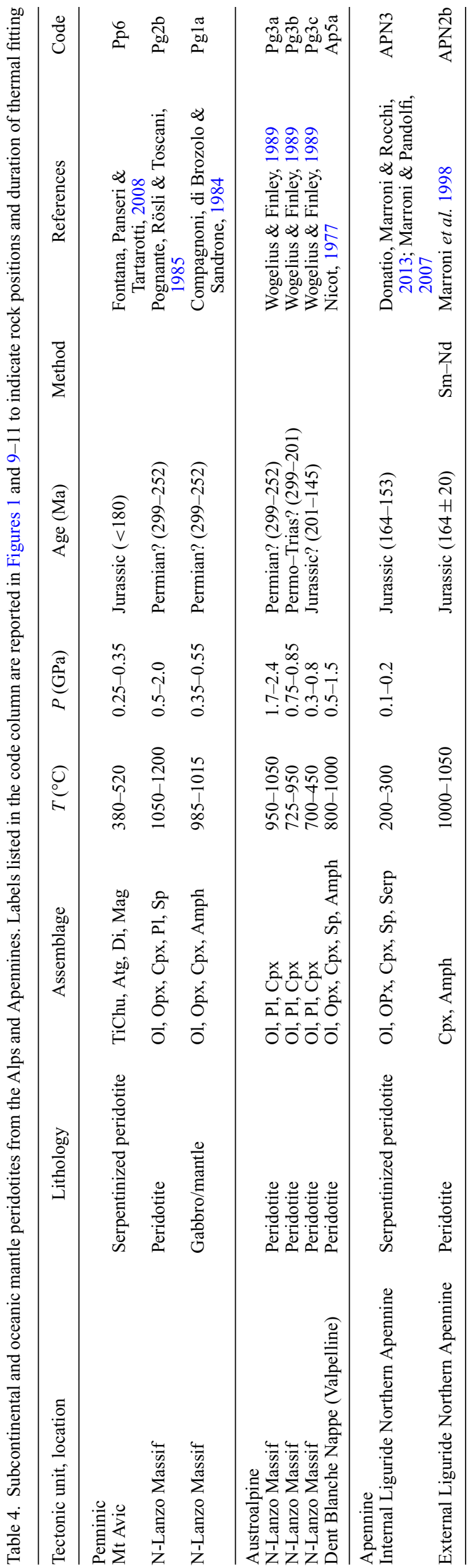

fabrics. The Penninic domain consists of mingled crustal slices deriving from both pre-Alpine continental and Mesozoic oceanic lithosphere, the latter tectonically sampled from the subducted Tethys Ocean (e.g. Platt 1986; Polino, Dal Piaz \& Gosso, 1990; Stöckhert \& Gerya, 2005; Malatesta et al. 2012; Roda, Spalla \& Marotta, 2012; Malatesta et al. 2013). In contrast, the Austroalpine domain does not contain Mesozoic ophiolites but is infolded within them and the related Mesozoic sediments all along its external boundary. Finally, the Helvetic domain consists of a Europe-verging thrust system that includes basement and cover slices structured during the late stages of the Alpine continental collision since Tertiary. Due to its shallow-level Alpine tectonic history, the Helvetic and Southalpine units (Fig. 1) broadly preserve preAlpine metamorphic, structural and stratigraphic imprints (Fig. 7), whereas Cretaceous-Paleocene Alpine high-pressure rocks are confined within the axial part of the chain in a rootless crustal prism consisting of Penninic and Austroalpine units. These latter are bounded by the Penninic frontal thrust (PF, Fig. 1) towards the European foreland and by the Periadriatic lineament (PL, Fig. 1) towards the Adriatic hinterland (Handy \& Oberhänsli, 2004; Thöni et al. 2008; Roda, Spalla \& Marotta, 2012). According to many authors the wide range of radiometric ages of high-pressure metamorphic imprints (Bousquet et al. 2004; Goffé et al. 2004; Handy et al. 2010; Lardeaux, 2014) suggests that they were buried and widely exhumed during subduction of the oceanic lithosphere (European lower plate), accompanied by tectonic erosion of the upper continental plate (Adria) before the onset of continental collision (e.g. Platt 1986; Polino, Dal Piaz \& Gosso, 1990; Spalla et al. 1996; Gerya \& Stöckhert 2005; Roda, Marotta \& Spalla, 2010; Roda, Spalla \& Marotta, 2012; Rubatto et al. 2011). Here, pre-Alpine structural, metamorphic and igneous relics are also preserved even if pervasive Alpine structural and metamorphic reworking shapes them into small-sized and scattered lenses, inhibiting the correlation of pre-Alpine structures at the regional scale.

\section{4.b. Lithology, structures, $P-T$ conditions and ages}

Asthenospheric upwelling associated with lithospheric thinning causes high thermal regimes in the thinned continental lithosphere (e.g. Thompson, 1981; England \& Thompson, 1984; Thompson \& England, 1984; Sandiford \& Powell, 1986; Spear \& Peacock, 1989; Beardsmore \& Cull, 2001), and in the Alps the only igneous and metamorphic effects indicating the occurrence of such a thermal state before the Alpine convergence are of Permian-Triassic age. They are detectable along the whole belt, from the Ligurian Sea to the Pannonian Basin, even in domains strongly reworked by the Alpine tectonics and metamorphism. These records consist of a widespread emplacement of Permian-Triassic basic to acidic igneous activity and huge gabbro bodies (Tables 2 and 3; Fig. 1) associated with regional 
Table 5. Ophiolitic gabbros from the Alps, Apennines and Corsica. The lack of $P-T$ estimates for Corsica gabbros inhibits their comparison with the model predictions. Labels listed in the code column are reported in Figures 1 and 9-11 to indicate rock positions and duration of thermal fitting

\begin{tabular}{|c|c|c|c|c|c|c|c|c|}
\hline Tectonic unit, location & Lithology & Assemblage & $T\left({ }^{\circ} \mathrm{C}\right)$ & $P(\mathrm{GPa})$ & Age (Ma) & Method & References & Code \\
\hline \multicolumn{9}{|l|}{ Penninic } \\
\hline Chennaillet & Troctolite & $\mathrm{Pl}, \mathrm{Cpx}, \mathrm{Ol}, \mathrm{Amph}$ & $700-800$ & $0.1-0.4$ & Jurassic (166-158) & $\mathrm{U}-\mathrm{Pb}$ & $\begin{array}{l}\text { Mevel, Caby \& Kienast, 1978; Li } \\
\text { et al. } 2013\end{array}$ & Pp5b \\
\hline Chennaillet & Troctolite & $\mathrm{Pl}, \mathrm{Cpx}, \mathrm{Ol}, \mathrm{Amph}$ & $710-940$ & $0.1-0.4$ & Jurassic (166-158) & $\mathrm{U}-\mathrm{Pb}$ & $\begin{array}{l}\text { Mevel, Caby \& Kienast, 1978; Li } \\
\text { et al. } 2013\end{array}$ & Pp5a \\
\hline S-Lanzo Massif & Gabbro-mantle & Ol, Opx, Cpx, Amph & $985-1015$ & $0.35-0.55$ & Jurassic (201-145) & & $\begin{array}{l}\text { Compagnoni, do Brozolo \& } \\
\text { Sandrone, } 1984\end{array}$ & $\operatorname{Pg} 1 b$ \\
\hline S-Lanzo Massif & Gabbro & $\mathrm{Pl}, \mathrm{Ol}, \mathrm{Cpx}$ & $750-900$ & $0.1-0.3$ & Jurassic (201-145) & & Pognante, Rösli \& Toscani, 1985 & $\operatorname{Pg} 2 \mathrm{a}$ \\
\hline Lanzo Massif (whole) & Gabbro & $\mathrm{Pl}, \mathrm{Cpx}, \mathrm{Ol}, \mathrm{Amph}$ & $800-850$ & & Jurassic (164-156) & $\mathrm{U}-\mathrm{Pb}$ & $\begin{array}{l}\text { Kaczmarek, Müntener \& Rubatto, } \\
2008\end{array}$ & $\mathrm{Pg} 4$ \\
\hline Voltri Massif & Gabbro & $\mathrm{Ol}, \mathrm{Pl}, \mathrm{CPx}$ & & & Jurassic $(182 \pm 19)$ & $\mathrm{Sm}-\mathrm{Nd}$ & Rampone et al. 2014 & Pp6 \\
\hline \multicolumn{9}{|l|}{ Apennine } \\
\hline $\begin{array}{l}\text { East Ligurian ophiolites, } \\
\text { Northern Apennines }\end{array}$ & Gabbro & $\mathrm{Pl}, \mathrm{Ol}, \mathrm{Cpx}, \mathrm{Ilm}, \mathrm{Amph}, \mathrm{Opx}$ & $800-950$ & $0.3-0.6$ & Jurassic (185-161) & $\mathrm{ft}$ Zrn & Tribuzio, Riccardi \& Ottolini, 1995 & APN1a \\
\hline Apennine & Gabbro & $\mathrm{Pl}, \mathrm{CPx}, \mathrm{Ox}, \mathrm{Amph}$ & $800-970$ & $0.3-0.6$ & Jurassic $(<180)$ & & Riccardi, Tribuzio \& Caucia, 1994 & APN1b \\
\hline Apennine & Gabbro & $\mathrm{Pl}, \mathrm{CPx}, \mathrm{Ox}, \mathrm{Amph}$ & $730-660$ & $0.1-0.3$ & Jurassic $(<160)$ & & $\begin{array}{l}\text { Riccardi, Tribuzio \& Caucia, 1994; } \\
\text { Tribuzio, Riccardi \& Messiga, } \\
\text { 1997; Rebay, Riccardi \& Spalla, } \\
\text { 2015 }\end{array}$ & APN1c \\
\hline Apennine & Gabbro & $\mathrm{Pl}, \mathrm{CPx}, \mathrm{Ox}, \mathrm{Amph}$ & $200-300$ & $0.1-0.2$ & Jurassic $(<160)$ & & $\begin{array}{l}\text { Riccardi, Tribuzio \& Caucia, 1994; } \\
\text { Tribuzio, Riccardi \& Messiga, } \\
\text { 1997; Rebay, Riccardi \& Spalla, } \\
\text { 2015 }\end{array}$ & APN1d \\
\hline Northern Apennine & Gabbro & $\mathrm{Ol}, \mathrm{Pl}, \mathrm{CPx}$ & & & Jurassic (188-170) & $\mathrm{Sm}-\mathrm{Nd}$ & $\begin{array}{l}\text { Tribuzio, Thirwall \& Vannucci, } \\
2004\end{array}$ & APN2 \\
\hline Cecina Valley & Gabbro & $\mathrm{Ol}, \mathrm{Pl}, \mathrm{CPx}$ & & & Jurassic (183-157) & $\mathrm{SmNd}$ & $\begin{array}{l}\text { Tribuzio, Thirwall \& Vannucci, } \\
2004\end{array}$ & APN3 \\
\hline \multicolumn{9}{|l|}{ Corsica } \\
\hline Balagne upper nappe & Gabbro & Pl, Cpx, Amph, Ap, Mag, Zr & & & Jurassic $(159 \pm 2)$ & $\mathrm{U}-\mathrm{Pb}$ & Li et al. 2015 & $\mathrm{C} 1$ \\
\hline Balagne upper nappe & Gabbro & $\mathrm{Pl}, \mathrm{Cpx}, \mathrm{Amph}, \mathrm{Ap}, \mathrm{Mag}, \mathrm{Zr}$ & & & Jurassic $(169 \pm 3)$ & $\mathrm{U}-\mathrm{Pb}$ & Rossi et al. 2002 & $\mathrm{C} 1$ \\
\hline Inzecca & Albitite & $\mathrm{Pl}, \mathrm{Qtz}, \mathrm{Cpx}, \mathrm{Amph}$ & & & Jurassic $(161 \pm 3)$ & $\mathrm{U}-\mathrm{Pb}$ & Ohnenstetter et al. 1981 & $\mathrm{C} 2$ \\
\hline Monte Maggiore & Gabbro & $\mathrm{Pl}, \mathrm{Cpx}, \mathrm{Ol}, \mathrm{Opx}$ & & & Jurassic (172-149) & $\mathrm{Sm}-\mathrm{Nd}$ & $\begin{array}{l}\text { Rampone, Hofmann \& Raczek, } \\
2009\end{array}$ & $\mathrm{C} 3$ \\
\hline
\end{tabular}




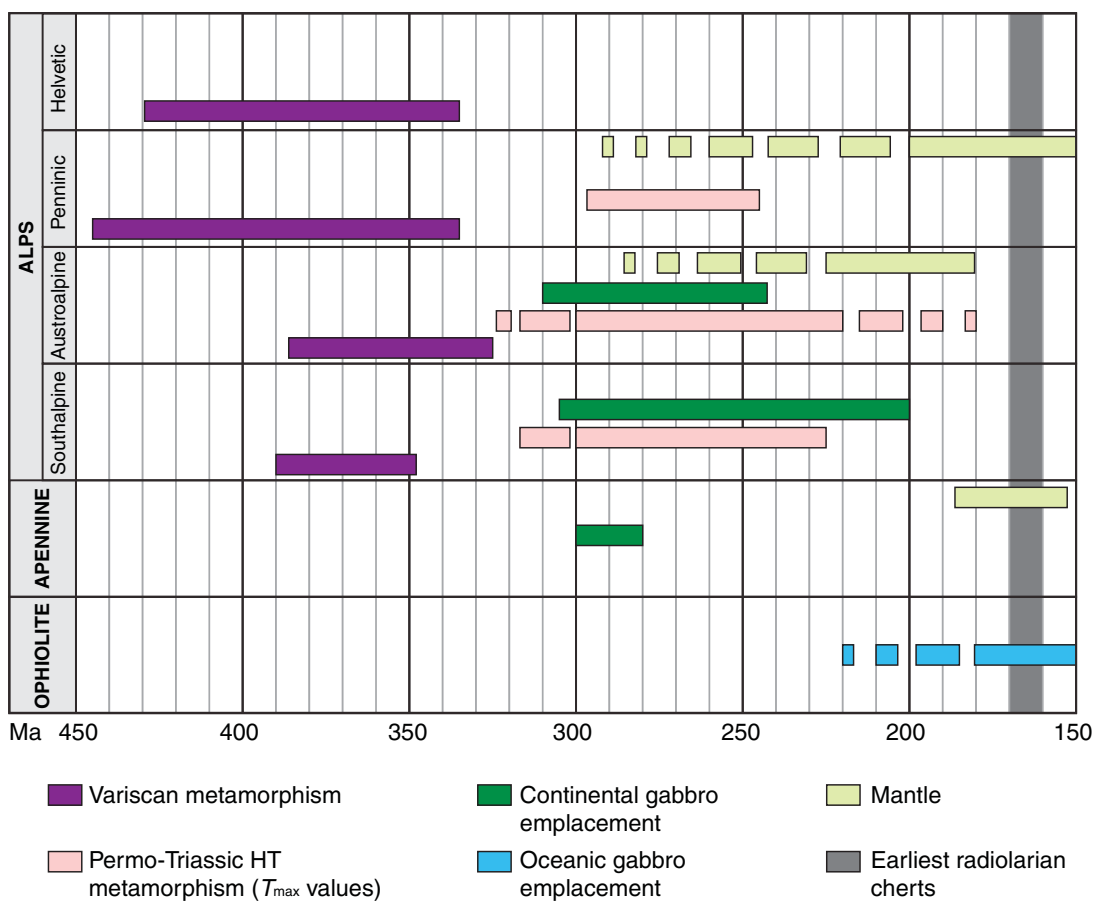

Figure 7. (Colour online) Timing of the metamorphic and magmatic events from Variscan to Jurassic time along the Alpine belt. Radiometric ages are plotted including analytical uncertainty intervals (dashed horizontal bars). Variscan evolutions are synthesized by data in Table 2. Permian-Triassic metamorphic and igneous data (continental gabbros) are synthesized by data in Table 3 and represented with their error margin. Ophiolites and mantle age data are listed in Tables 4 and 5 . The grey stripe indicates the age of the earliest radiolarian cherts related to Alpine ophiolites (Cordey \& Bailly, 2007).

high-temperature - low-pressure (HT-LP) metamorphism, which postdate structures and metamorphic imprints widely developed during the Variscan subduction and collision (Fig. 7). These features are frequently associated with subcontinental peridotites (Table 4) and are mainly confined to the Austroalpine and Southalpine domains (e.g. Lardeaux \& Spalla, 1991; Bonin et al. 1993; Bussy et al. 1998; Rottura et al. 1998; Schuster et al. 2001; Stahle et al. 2001; Rebay \& Spalla, 2001; Rampone, 2002; Peressini et al. 2007; Marotta, Spalla \& Gosso, 2009; Spalla et al. 2014).

Metamorphic Permian-Triassic imprints are widely recorded in the lower, intermediate and upper continental crust of the Austroalpine and Southalpine domains; only a few records have been recognized in the upper and intermediate Penninic crust of Western Alps, and they are never detected in the Helvetic domain (Fig. 1). In the Penninic domain, HT assemblages mainly developed in sillimanite-bearing metapelites and metaintrusives. They are generally derived from re-equilibration under low-pressure conditions and interpreted as Permian in age (Bouffette, Lardeaux \& Caron, 1993; Table 2). Sapphirine-bearing HT- to UHT-IP granulites of the Gruf Complex have been recently petrologically and chronologically investigated, revealing an age of 260-290 Ma for $T_{\max }$ conditions (Galli et al. 2011). The exhumation of Penninic HT Permian-Triassic rocks was generally associated both with cooling and heating (Desmons, 1992; Bouffette, Lardeaux \& Caron, 1993) and occurred before the Alpine convergence, whereas the exhumation of UHT Gruf granulites occurred at the end of the Alpine convergence from the base of the internal European passive margin where they lay since the Permian lithospheric thinning (Galli et al. 2011). In the Austroalpine domain, Permian-Triassic HT metamorphism mainly developed in sillimanite- and biotite-bearing gneisses; it is associated with minor mafic granulites, amphibolites and high-grade marbles (Table 2). HT minerals locally mark mylonitic fabrics within discrete shear zones (Lardeaux \& Spalla 1991; Spalla et al. 1991), and exhumation paths can be characterized by cooling, heating or isothermal decompression (e.g. Dal Piaz, Lombardo \& Gosso, 1983; Stöckhert, 1987; Vuichard, 1987; Lardeaux \& Spalla, 1991; Spalla, Messiga \& Gosso, 1995; Schuster et al. 2001; Manzotti \& Zucali, 2013). Locally, the exhumation was accomplished up to very shallow structural levels (e.g. Rebay \& Spalla, 2001), suggesting that some Austroalpine units belonged to a thinned continental crust before being subducted during Cretaceous convergence. In the Southalpine basement, Permian-Triassic HT metamorphism developed in metapelites, mafic granulites, amphibolites and high-grade marbles and mainly re-equilibrated under granulite-amphibolite-facies conditions (Table 2). HT paragenesis marks a pervasive foliation that is locally mylonitic within up to kilometre-thick discrete shear zones that are often steepened by Alpine thrusting (e.g. Bertotti et al. 1993; Gosso, Siletto \& Spalla, 1997). Mylonitic belts developed under upper amphibolitegranulite- to greenschist-facies conditions are widespread in the central Southalpine domain, accounting for regional-scale pervasive extensional tectonics (Brodie, Rex \& Rutter, 1989; Diella, Spalla \& Tunesi, 
1992; Bertotti et al. 1993) and widely interpreted as related to regional-scale normal faults responsible for the exhumation of HT-LP complexes (e.g. Brodie, Rex \& Rutter, 1989; Handy et al. 1999) during a continuous evolution from Permian to Triassic or Jurassic time (e.g. Bertotti et al. 1993). Intrusive stocks emplaced at shallow levels during Permian - Early Triassic time are associated with metamorphic aureoles (Povoden, Horacek \& Abart, 2002; Benciolini et al. 2006; Gallien, Abart \& Wyhlidal, 2007). Exhumation paths were characterized by cooling or by increasing temperature during decompression (e.g. Brodie, Rex \& Rutter, 1989; di Paola \& Spalla 2000) and were generally accomplished under a high thermal regime. HT PermianTriassic mineral associations have never been detected in the pebbles of Permian conglomerates from the Orobic Alps, suggesting that HT rocks were not yet exposed in their Variscan source areas before the late Permian - Triassic period (Spalla et al. 2009; Zanoni, Spalla \& Gosso, 2010).

Permian-Triassic continental gabbros have been detected in the Austroalpine and Southalpine domains of the Alps (Bonin et al. 1993; Rottura et al. 1998; Stahle et al. 2001; Spiess et al. 2010; Spalla et al. 2014). The mafic products of the widespread PermianTriassic igneous activity mainly consist of gabbroic bodies with subcontinental peridotites (Brodie, Rex \& Rutter, 1989; Bonin et al. 1993; Schuster et al. 2001; Stahle et al. 2001; Rampone 2002; Spalla et al. 2014). They occurred at different structural levels, and the country rocks vary from high-temperature intermediate-pressure metamorphics to Triassic carbonatic sediments (Sills 1984; Handy \& Zingg 1991; Lardeaux \& Spalla 1991; Gallien, Abart \& Wyhlidal, 2007; Miller et al. 2011; Spalla et al. 2014). From a geochemical point of view most of the gabbros have a tholeiitic to alkaline signature, although they are generally considered to be generated from variably contaminated mantle sources in an extensional tectonic regime under a high thermal state associated with lithospheric thinning and rifting (Spalla et al. 2014 and references in Table 3). The ages of these gabbroic intrusions in the Austroalpine domain cluster around Permian (Table 3). The main Triassic magmatic signal is recorded in the Southalpine domain by Predazzo and Monzoni intrusives and in the Ivrea Zone by alkaline rocks (Table 3). Stahle et al. (2001) interpret this igneous activity as the result of a long-lasting process of active rifting.

Few Permian-Triassic continental mantle slices have been found in the Alps (Table 4). In particular, the North Lanzo body in the Penninic domain is characterized by subcontinental lithospheric mantle protoliths and underwent progressive exhumation during preoceanic lithosphere extension and rifting. The South Lanzo body shows impregnation by mid-ocean-ridge basalt (MORB) melts rising from the underlying molten asthenosphere during the rifting stage of the LiguriaPiemonte Ocean (Piccardo \& Guarnieri 2010). The Lanzo peridotites would therefore represent a lithosphere changing from a thinned continental plate to an ocean-continent transition zone (OCTZ) (Piccardo \& Guarnieri 2010). In the Austroalpine domain, a small peridotite body has been detected in the Dent-Blanche Nappe (Nicot, 1977). Although no radiometric age is available for this mantle rock, its structural relation with continental rocks of the Valpelline Series and the analogy with similar rocks of the Ivrea Zone suggest a Permian age and therefore a continental affinity for this peridotite.

In the Alps, the transition from rifting to oceanic spreading is indicated by the deposition of post-rift sediments (172-165 Ma, Baumgartner et al. 1995; Stampfli et al. 1998; Bill et al. 2001; Handy et al. 2010) postdating syn-rift Triassic deposits (e.g. Gillcrist, Coward \& Mugnier, 1987). This transition involved the exhumation and serpentinization of the subcontinental mantle at the Liguria-Piemonte Ocean margins (e.g. Desmurs, Manatschal \& Bernoulli, 2001; Manatschal, 2004; Manatschal \& Müntener, 2009). The radiometric ages of the ophiolitic gabbros (Fig. 7, Table 5) clustering around approximately $160 \mathrm{Ma}$ (Mevel, Caby \& Kienast, 1978; Li et al. 2013), with older values of 166 $183 \mathrm{Ma}$ from the Apennines, Corsica and Erro-Tobbio ophiolitic units (e.g. Tribuzio, Thirwall \& Vannucci, 2004; Rampone et al. 2014; Li et al. 2015), confine the beginning of the spreading of the Liguria-Piemonte Ocean. Ages of $198 \pm 22$ (Sm-Nd on gabbro WR) have been obtained by Costa \& Caby (2001) in ophiolites from the Western Alps (Chenaillet) and interpreted by the authors as the signature of lithospheric extension announcing the oceanic spreading. Oceanic gabbros exclusively occur in the ophiolitic sequences of the Penninic domain (Fig. 1). They generally have a MORB affinity and are usually associated with serpentinized mantle but sometimes to volcanic sequences, such as lava flows, pillow basalts and pillow breccias, and oceanic sediments (e.g. Mevel, Caby \& Kienast, 1978; Ohnenstetter et al. 1981; Riccardi, Tribuzio \& Caucia, 1994; Martin, Tartarotti \& Dal Piaz Giorgio, 1994).

Oceanic mantle rocks coming from the Penninic domain (Table 4) are variably serpentinized peridotites. Based on the relict texture, mineralogy and structural relations with gabbros and rodingites, the serpentinized peridotite of Mt Avic (Table 4) is considered to have an oceanic affinity (Fontana, Panseri \& Tartarotti, 2008). Although the gabbroic bodies of the Lanzo Massif are considered to have originated during the opening of the Jurassic-Piedmont Ligurian ocean (Lagabrielle, Fudral \& Kienast, 1989; Pognante, Rösli \& Toscani, 1985 ) or during the earliest stages of the formation of the embryonic oceanic crust (Kaczmarek, Müntener \& Rubatto, 2008), the associated peridotites show a more complex affinity as already discussed. The Mg-rich gabbroic rocks of Erro-Tobbio complex in Voltri Massif (Ligurian Alps) is considered as representative of syn-rift melt intrusions in thinned lithospheric mantle exhumed at ocean-continent transition domains (Rampone et al. 2014). They represent the oldest gabbroic bodies of the Alpine Tethys (201-163 Ma, Rampone et al. 2014). Because rocks from the Alps 


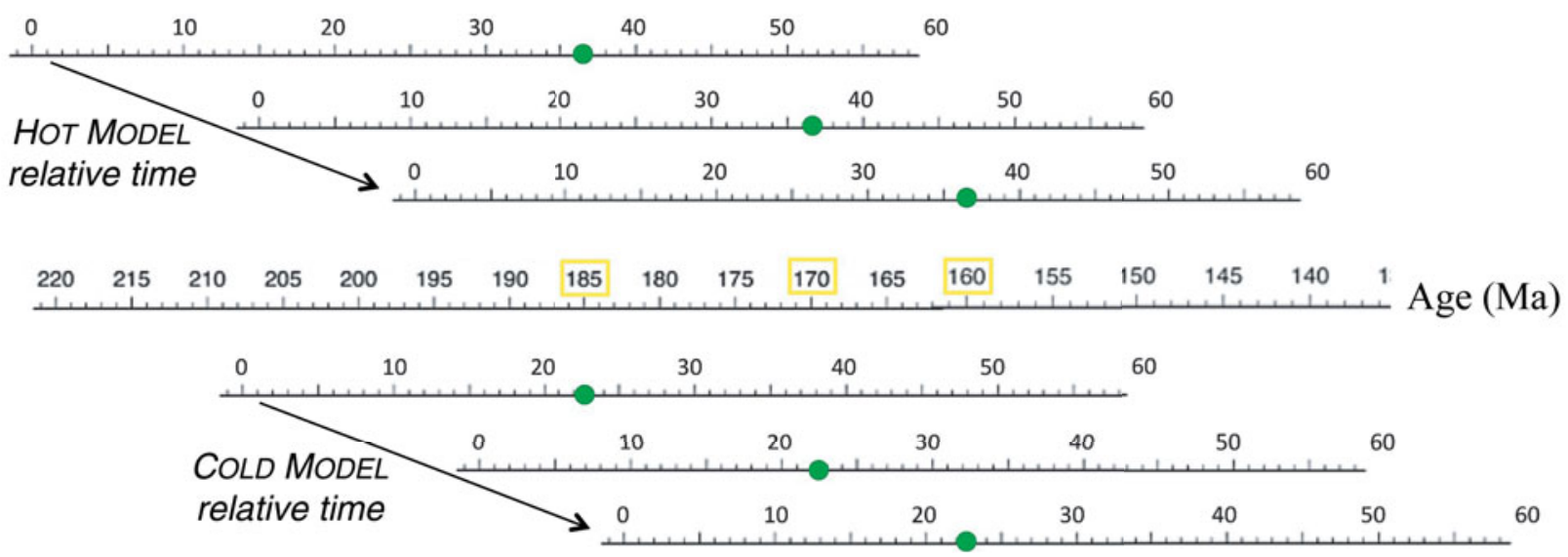

Figure 8. (Colour online) Time references from the hot and cold models with respect to the age of the oldest gabbros (160 Ma, $170 \mathrm{Ma}$ and $185 \mathrm{Ma}$ ), based on the literature for the Northern Apennines and Western Alps (Tribuzio, Riccardi \& Ottolini, 1995; Li et al. 2013; Rampone et al. 2014). Green circles identify the times when the mantle partial melting occurs in the hot model (36.4 Ma) and in the cold model (22.4 Ma).

are widely deformed and metamorphosed during Alpine subduction and collision, we add ophiolites from the Northern Apennines that escaped pervasive subduction-related metamorphic re-equilibration and the unique case of mafic granulite from the continental crust to the collection of natural data (Tables 3-5). The Northern Apennines are characterized by oceanic and continental units (Fig. 1). Oceanic units are divided into two different groups of thrust nappes - the Internal and External Ligurian units (e.g. Marroni \& Pandolfi, 2007) - strongly deformed under low-grade metamorphic conditions (Marroni \& Pandolfi, 2007; Donatio, Marroni \& Rocchi, 2013). An ophiolitic sequence of Jurassic age and a sedimentary cover ranging in age from Late Jurassic to Paleocene characterize the Internal Ligurian units (Marroni \& Pandolfi, 2007). In the External Ligurian units, Late Cretaceous sedimentary melanges containing slide-blocks of ophiolites occur at the base of the Upper Cretaceous carbonate turbidites (Helminthoid Flysch; Marroni \& Pandolfi, 2007). The Ligurian units were thrust onto the Tuscan nappes during the Oligo-Miocene post-collisional convergence. The successions of the Tuscan units belong to the Adria passive continental margin, recording in sequence rifting-, cooling- and subsidence-related imprints during the opening of the Liguria-Piemonte Ocean (Marroni \& Pandolfi, 2007). Underlying Tertiary metamorphic units are exposed in rare tectonic windows (Fig. 1).

Granulitic gabbros of the External Liguride Unit associated with felsic granulites locally intrude mantle peridotites (Marroni \& Tribuzio, 1996; Marroni et al. 1998). The emplacement of the gabbroic protoliths occurs at deep crustal levels of late Carboniferous - early Permian age (approximately $290 \mathrm{Ma}$ ), and their country rocks are felsic granulites of the lower continental crust. Most likely in association with the subcontinental mantle, mafic and felsic granulites underwent a multistage exhumation beginning during PermianTriassic time and ending during Late Triassic - Middle
Jurassic time, when they were finally exhumed to shallow levels by extensive brittle faulting (Marroni et al. 1998). The External Liguride Unit is interpreted as an ocean-continent transition zone (Marroni et al. 1998).

Some Jurassic oceanic gabbros have been detected in the Apennines (Table 5; Riccardi, Tribuzio \& Caucia, 1994; Tribuzio, Riccardi \& Ottolini, 1995; Tribuzio, Riccardi \& Messiga, 1997; Rebay, Riccardi \& Spalla, 2015). The oldest gabbro bodies (169-179 Ma) belong to the External Liguride Unit in the Northern Apennines. Despite their N-MORB affinity, Tribuzio, Thirwall \& Vannucci (2004) interpreted these gabbros as having developed during an intermediate stage of the rifting process that led to the opening of the Ligurian Tethys and not strictly related to the oceanic spreading.

The peridotite of the External Liguride Unit has been interpreted as the mantle of an ocean-continent transition zone (Marroni et al. 1998), while the serpentinized peridotite of the Internal Liguride Unit is suggested to be representative of the Jurassic oceanic lithosphere of the Liguria-Piemonte Ocean (Marroni \& Pandolfi 2007; Donatio, Marroni \& Rocchi, 2013).

\section{Comparison between the model predictions and the natural data}

Before proceeding with the comparison between the model predictions and the natural data, it is necessary to reference the relative time of the numerical simulation with respect to the natural ages. To achieve this goal, we decided to match the beginning of the mantle partial melting in the model with the ages of the gabbros available in the literature (Table 5). In particular, we chose the three absolute ages of $160 \mathrm{Ma}, 170 \mathrm{Ma}$ and $185 \mathrm{Ma}$ interpreted by different authors (Tribuzio, Riccardi \& Ottolini, 1995; Tribuzio, Thirwall \& Vannucci, 2004; Li et al. 2013, 2015) as the oldest gabbro ages of Liguria-Piemonte Ocean; they can therefore be considered the temporal markers of the early oceanic spreading. Figure 8 depicts the time referencing of 
both models with respect to the three chosen absolute ages.

The data from the continental crust (Tables 2, 3) are compared with the continental markers; the data from the mantle (Table 4) are compared with the dry or serpentinized mantle according to the estimated $P-T$ conditions and rock assemblages. In the following, the process starting from the exhumation of serpentinized mantle and proceeding through the oceanic spreading is referred to as 'oceanization'. The oceanic spreading leading to the formation of the LiguriaPiemonte Ocean starts when the conditions favourable for mantle partial melting are attained in the system. We compare the pressure-temperature values predicted for markers belonging to the oceanic lithosphere with the $P-T$ estimates available for gabbros belonging to Alpine and Apennine ophiolitic complexes (Table 5), as well as those available for the oceanic mantle (Table 4).

To verify if the simulated geodynamic context can reproduce a thermal state that is compatible with that recorded by the lithosphere during the Permian-Triassic period, in Figure 9 we show the duration of the agreement between the predictions and the natural data for the three different absolute ophiolite ages in terms of lithology and coincident $P-T$ values compared to the radiometric (black thick segments) and geologic (grey thick segments) ages of the natural data. In the following discussion, we refer to a 'complete fit' when there is agreement between the model predictions and the natural data in the lithology, $P-T$ values and ages. The fit is considered 'partial' if age coincidence is lacking. The comparative analysis takes into account the natural data with their error margins (Tables 2-5).

The predictions of both the hot and cold models show complete fits with the same data, although the number of fitting markers is in general lower in the cold model than in the hot model. Complete fits are realized with a maximum of 13 data points out of the available 44: 10 are of the oceanic lithosphere type (Pp5a, Pp5b, Pp6, Pg1b and Pg2a, Penninic domain; and APN1a, APN1b, APN1c, APN1d and APN3, Apennine domain), and 2 are of the pre-oceanic lithosphere type (Ap5a, Pg3b and Pg3c, Austroalpine domain). Good agreement is obtained for all of the oceanic lithosphere type markers with the natural data from the Alpine and Apennine ophiolites for all of the ages proposed in the literature.

The predictions do not show a complete fit with the continental crust data. Nevertheless, the hot model reproduces thermo-barometric conditions that are compatible with those of most of the continental crust data in the interval during 220-150 Ma, according to the time of the oldest ophiolitic ages. A similar situation holds for the cold model, although with a very low number of markers. However, the radiometric ages of these data are older than the oldest model predictions.

Some data, that is, Sp1 (Eisacktal), Sp3 (DervioOlgiasca) and $\mathrm{Sp5a}$ and $\mathrm{Sp5b}$ (Ivrea) which are from the Southalpine domain, Ap17 (Sopron) and Ap7 and Ap8 (Valpelline) which are from the Austroalpine domain and Pp4 (Gruf) which is from the Penninic domain, do not show any agreement, even thermo-barometric, because the estimated temperatures are higher than the predicted temperatures.

Figures 10 and 11 depict the spatial distributions of the markers that exhibit complete fits with the data for the hot and the cold models, respectively. With the exception of the Pg3c and Ap5a (continental mantle, Austroalpine domain) data, the $P-T$ conditions that are compatible with those of the other natural data are predicted only in a $200 \mathrm{~km}$ wide region centred at the ridge and only after the formation of the oceanic lithosphere (Figs 10d, e, 11d, e). The Pg3c datum fits the mantle-type markers at shallow structural levels only after a significant thermo-mechanical thinning of the lithosphere. This occurs after approximately 5.4 Ma in the hot model, affecting a large amount of the continental lithospheric mantle (Fig. 10b). In the cold model, the fitting starts very early and affects only a $60 \mathrm{~km}$ wide area around the future ridge, concurrently with the thermal thinning localization. For both models, the fitting persists until the late stages of the evolution and affects the shallow structural levels of the entire nonserpentinized lithosphere. The hot model predicts $P-T$ conditions that are compatible with the Ap5a datum (continental mantle, Austroalpine domain) starting at the beginning of the simulation (Fig. 10a), while in the cold model the favourable conditions occur only after significant heating of the system (Fig. 11b). This result supports the idea that Ap5a is a representative slice of the continental mantle under the perturbed $P-T$ conditions rather than under the thermal regime of a stable lithosphere. Pglb and Pg2a, both of which belong to the gabbroic rocks of the Lanzo Massif for both the hot and cold models, exhibit a complete fit with markers of the oceanic lithosphere type but at different structural levels. Pg1b agrees at deeper structural levels (Figs 10d, 11d), consistent with a gabbroic melt impregnation of the lithosphere (Compagnoni, di Brozolo \& Sandrone, 1984). Instead, Pg2a agrees at shallower levels (Figs 10d, 11d), in agreement with a decompression stage of an older subcontinental lithospheric mantle under hydrated conditions (Pognante, Rösli \& Toscani, 1985). Fits with Pp6 (Mont Avic serpentinized peridotite), APN1a (gabbros of North Apennines ophiolites) and APN1b (gabbros of Apennines Ophiolites) data start at the beginning of the oceanization (Figs 10d, 11d) and can be compatible with either the oceanic lithosphere or the ocean-continent transition zone. During oceanic spreading, the complete fit localizes at the marginal portions of the oceanic basin (Figs 10d, e, 11d, e). Finally, the complete fit with the APN1c, APN1d and APN3 (gabbros and mantle of Apennines ophiolites) and Pp5a and Pp5b (Chenaillet) data occurs during oceanic spreading and at the appropriate litho-structural levels of the oceanic lithosphere (Figs 10e, 11e). 


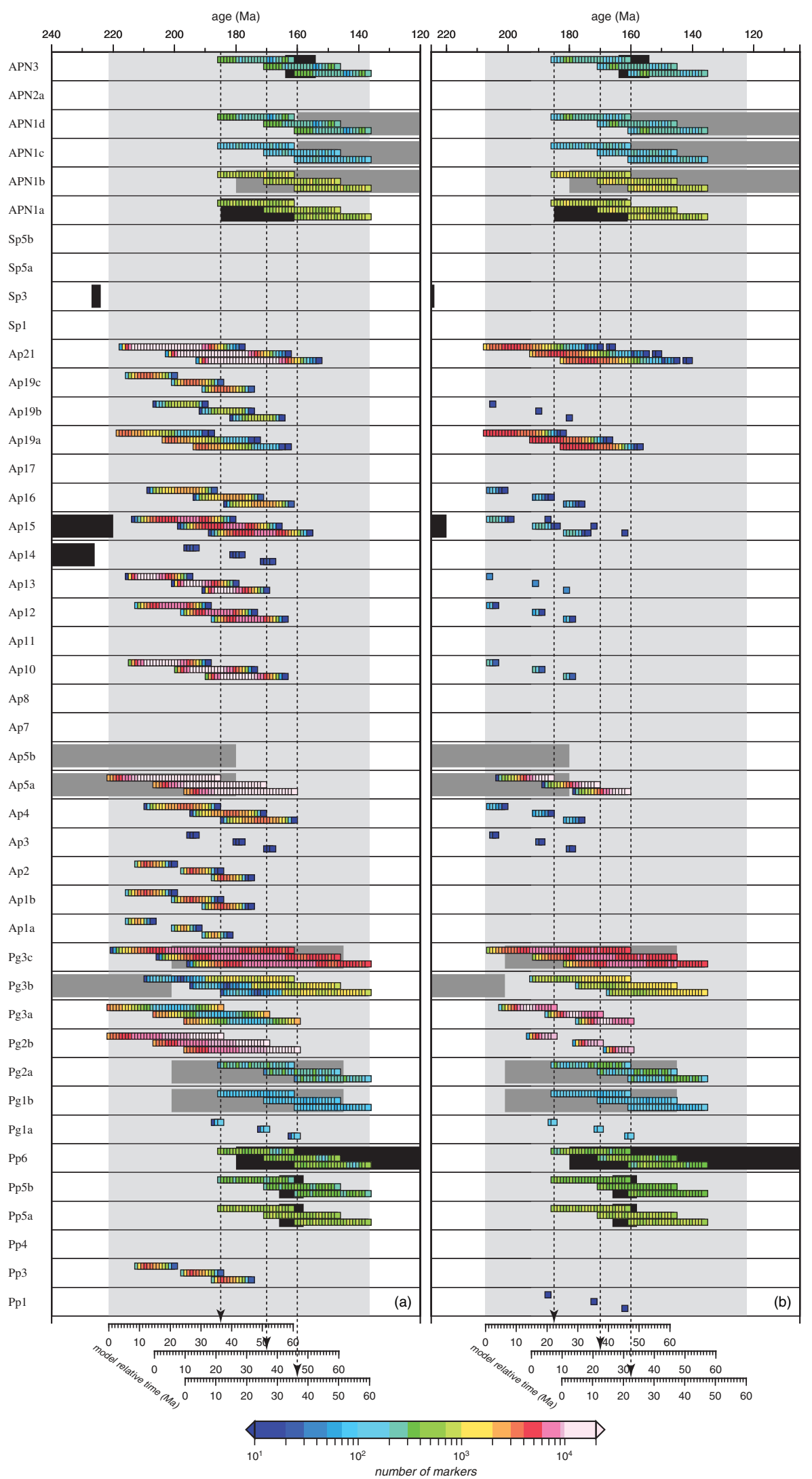

Figure 9. (Colour online) Duration of the agreement between the predictions and the natural data as well as number of fitting markers (colours) for the three different ages of the oldest gabbros (160 Ma, $170 \mathrm{Ma}$ and $185 \mathrm{Ma}$, dashed black lines) in terms of lithological affinity and coincident $P-T$ values compared to the radiometric (black thick segments) and geologic (grey thick segments) ages of the natural data. In the text, we refer to the result as a 'complete fit' when the model predictions and the natural data of the lithological affinity, $P-T$ values and ages agree. The fit is considered 'partial' if age agreement is lacking. Panel (a) refers to the hot model, whereas panel (b) refers to the cold model. The light grey area represents the duration of the numerical simulation. The codes are defined in Tables 2-5. 

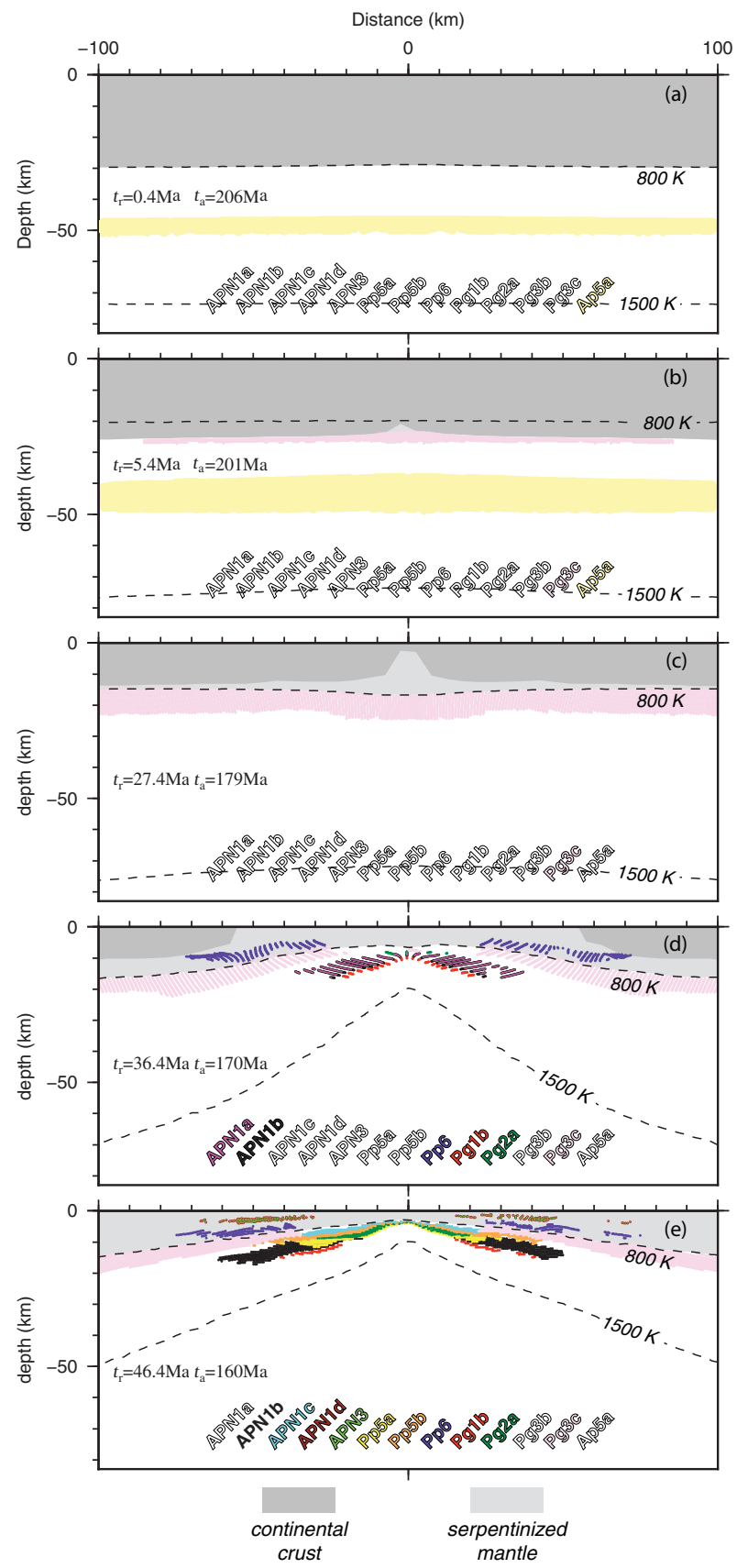

Figure 10. (Colour online) Spatial distribution of the markers that guarantee a complete fit of the data for the hot model at different stages of the evolution. The dashed black lines indicate the $800 \mathrm{~K}$ and $1500 \mathrm{~K}$ isotherms. The colours identify the markers showing a complete fit with the natural data as specified in the legend; $t_{\mathrm{r}}$ indicates the time span from the beginning of the simulation and $t_{\mathrm{a}}$ indicates the absolute age constrained choosing the oldest gabbro age at $170 \mathrm{Ma}$ (see discussion in the text). The codes are defined in Tables 2-5.

\section{Discussion}

The model of crustal extension presented here, characterized by a weak lower crust and mantle serpentinization, results in symmetric rifting of the continental lithosphere and exhumation of a serpentinized lithospheric mantle. Our results support the idea that the occurrence of serpentinization of the mantle can favour the exhumation of the lithospheric mantle before

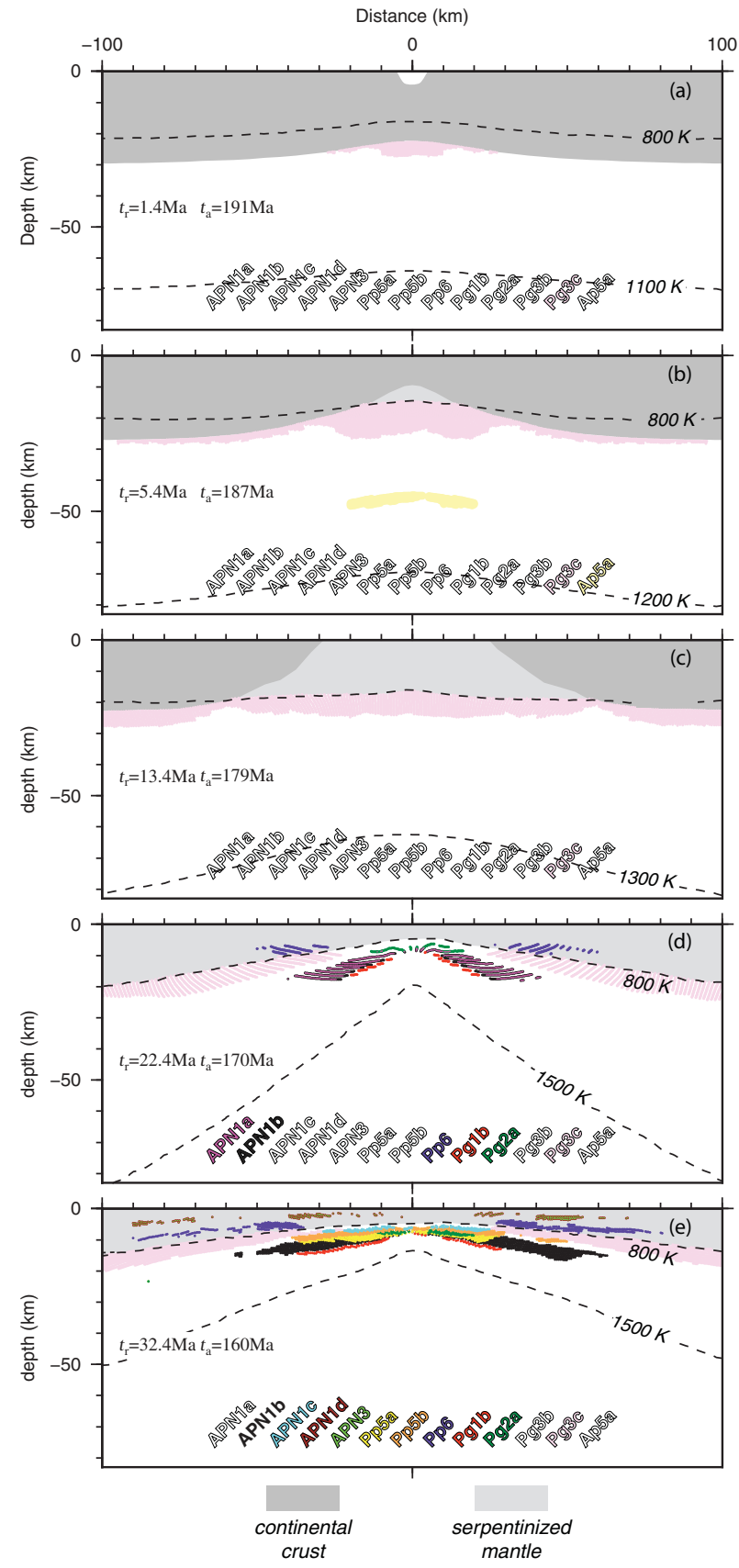

Figure 11. (Colour online) Spatial distribution of the markers that guarantee a complete fit of the data for the cold model at different stages of the evolution. The dashed black lines indicate the $800 \mathrm{~K}$ and $1500 \mathrm{~K}$ isotherms. The colours identify the markers showing a complete fit with the natural data specified in the legend; $t_{\mathrm{r}}$ indicates the time span from the beginning of the simulation and $t_{\mathrm{a}}$ indicates the absolute age constrained choosing the oldest gabbro age at $170 \mathrm{Ma}$ (see discussion in the text). The codes are defined in Tables 2-5.

the oceanic spreading in agreement with Lagabrielle \& Cannat (1990) and Pérez-Gussinyé et al. (2006).

The onset of lithospheric thinning localized around the future ridge strongly depends on the initial lithosphere thermal state: for a cold and strong lithosphere, the thinning is very rapid (after approximately $4.4 \mathrm{Ma}$ ) with respect to a hot and weak lithosphere (after approximately $15.4 \mathrm{Ma}$ ). Similarly, the time span between 
the onset of thinning and the occurrence of crustal break-up is shorter for a cold lithosphere (approximately $3 \mathrm{Ma}$ ) than for a hot lithosphere (approximately $16 \mathrm{Ma}$ ). These dynamics are attributable to the concurrent roles of the prescribed far-field traction and mantle upwelling flow. In the hot model, the contribution of the upwelling mantle flow to the lithosphere extension becomes efficient only in the advanced stages of the evolution, after the onset of the mantle partial melting. In contrast, for the cold model, both forces concur to the extension dynamics from the early stages of the evolution. These results agree with the models by Brune \& Autin (2013) and Manatschal, Lavier \& Chenin (2015) in which the break-up of a hotter and weaker lithosphere occurs later than in a colder and stronger lithosphere. In the case of a higher thermal state of the pre-rifting lithosphere, the viscous crustal layer is thicker than the brittle portion; consequently, the brittle strain softening is less efficient at focusing the deformation into discrete shear zones (Brune \& Autin, 2013). Lavier \& Manatschal (2006) and van Avendonk et al. (2009) suggest the opposite behaviour when the strong gabbroic lower crust is taken into account. In their models, a cold and strong lithosphere results in a longer rifting duration. On the other hand, the occurrence of a strong lower crust for the Alpine pre-rifting lithosphere is in contrast to the lithostratigraphy of the pre-Alpine continental crust. Given the number and size of PermianTriassic gabbroic intrusions in the Alpine crust, the amount of detectable gabbroic rocks is less than $5 \%$ of the total pre-Alpine lower continental crust actually exposed along the whole Alpine belt, which can be estimated from the tectonic map of the Alps (Bigi et al. 1990; Schmid et al. 2004) taking into account both units deeply involved in or escaping the Alpine subduction. Even considering a lower crust of the pristine passive margin that is richer in gabbroic rocks, it is reasonable to predict that, during the Alpine convergence, a selective tectonic sampling of the gabbro-poor lower crust does not occur. A coherent gabbroic lower crust for the Alpine pre-rifting lithosphere therefore seems unlikely.

For both chosen initial thermal configurations of the lithosphere, the exhumation of the serpentinized mantle starts before the oceanic spreading and the mantle partial melting (considered in our study coincident with gabbros formation), making the model compatible with the magma-poor rifting suggested for the Alpine case (e.g. Lavier \& Manatschal, 2006; Pérez-Gussinyé et al. 2006; Manatschal \& Müntener, 2009; Manatschal, Lavier \& Chenin 2015), developing an ocean-continent transition zone similar to Galizia margin (e.g. Boillot, Girardeau \& Kornprobst, 1989; Manatschal, 2004; Hébert et al. 2008). The exhumation of a serpentinized lithospheric mantle before the oceanic spreading of the Liguria-Piemonte Ocean is also suggested based on the geochemical analysis of the syn-rift Alpine crust and sediments (Pinto et al. 2015).

For both the hot and cold models, the mantle partial melting does not appear immediately after the crustal break-up but after approximately $5 \mathrm{Ma}$ in the hot model and after approximately $15 \mathrm{Ma}$ in the cold model. This result is dependent on the thermal field predicted when the crustal break-up occurs. The hot model predicts more suitable thermo-barometric conditions for mantle melting a few million years after the crustal break-up. In contrast, in the cold model a long time is required to increase the thermal state to satisfy the pressure-temperature conditions that are suitable for mantle melting. Despite the different partial timings of each stage in the two models, once the serpentinization starts and the deformation localizes around the future ridge, the time required for the mantle partial melting and the beginning of oceanic spreading is comparable in the two models: $18 \mathrm{Ma}$ for the cold model and $21 \mathrm{Ma}$ for the hot model.

The continental crust thickness sensibly decreases during the extension but with different rates. In the hot model, the crustal thickness decreases from $30 \mathrm{~km}$ to approximately $18 \mathrm{~km}$ at the margin and to approximately $5 \mathrm{~km}$ close to the OCTZ within $31.4 \mathrm{Ma}$ after the beginning of the evolution. In the cold model, it decreases from $30 \mathrm{~km}$ to approximately $22 \mathrm{~km}$ at the margin and to approximately $20 \mathrm{~km}$ close to the OCTZ within 21.4 Ma after the beginning of the evolution. Afterwards, in both models no further significant thinning occurs within the continental crust. The hyperextended margin envisaged by the hot model satisfies the model of an Alpine Tethys hyperextended system (Manatschal, Lavier \& Chenin, 2015). Our results indicate that in the proximity of the ridge, for both models, the extension rate at the base of the crust increases by a factor of 2-4 from the continental break-up to oceanization, in agreement with Whitmarsh, Manatschal \& Minshull (2001). Furthermore, at the advanced stages of the extension after the beginning of oceanic spreading, the extension rate may overcome the far-field traction.

A thinned continental crust (passive margin), an ocean-continent transition zone and an oceanic lithosphere characterize the final structure of the system from the periphery to the centre of the model domain for both thermal states. Our results show that the formation of the OCTZ starts 5-15 Ma before the partial melting of the mantle and develops with a size ranging over $160-280 \mathrm{~km}$ (according to the initial thermal configuration of the lithosphere), which is compatible with the observations of Galicia Margin (e.g. Boillot, Beslier \& Girardeau, 1995; Hébert et al. 2008) and similar to the interpretation for the Alpine rift (Manatschal \& Müntener, 2009). This suggests that if the estimate of the oceanic basin width is based simply on the coincidence between the continental crust breakup and the onset of gabbros emplacement, as stated in several models proposed for the Alpine domain (e.g. Li et al. 2013), the effective basin width will be underestimated. In particular, Li et al. (2013) estimated a basin width of $300 \mathrm{~km}$ after $10 \mathrm{Ma}$ of extension at a full spreading rate of $3 \mathrm{~cm} \mathrm{a}^{-1}$. In contrast, our model indicates that the extension of the basin would range 
over $360-480 \mathrm{~km}$ after the same time of $10 \mathrm{Ma}$ for a full extension rate of $2.5 \mathrm{~cm} \mathrm{a}^{-1}$.

The comparison between the natural data and the model predictions shows good agreement with all of the oceanic data for both the hot and cold models. In contrast, the comparison with the data from the continental crust lacks a complete fit because ages are not coincident. The lithological and thermal fits predicted by the hot and cold models with significant delays (from the Permian-Triassic to the Late Triassic - Late Jurassic periods; Figs 7, 9) suggest that, according to both models, the effects of a positive thermal anomaly should be recorded in the continental crust of both passive margins at 220-150 Ma. These effects have not yet been detected in the pre-Alpine continental crust of the Alps and Apennines. Similarly, the mantle partial melting occurs in both models $36.4 \mathrm{Ma}$ and 22.4 Ma after the beginning of the extension in the hot and cold models, respectively, that would correspond to the early gabbros at $185 \mathrm{Ma}$, which is significantly younger than the Permian-Triassic continental gabbro emplacements. This time misfit supports the interpretation predicting a thermo-mechanical perturbation of the continental lithosphere in this portion of the Tethys due to the Variscan collision and the late orogenic extension (Spalla et al. 2014). In addition, the more favourable thermal regime predicted by the hot model could be due to a previously perturbed system either by the Variscan orogenic collapse or by an already thermally eroded and softened lithosphere. However, the sole orogenic collapse mechanism is not sufficient to reproduce the thermo-barometric conditions of the HT-LP Permian-Triassic metamorphism and intense igneous activity recorded in the continental lithosphere (Marotta \& Spalla, 2007; Marotta, Spalla \& Gosso, 2009).

Finally, although the symmetry of the predicted thermal anomaly around the future ridge, HT natural parageneses from the Permian-Triassic continental lithosphere of the Alps are concentrated in the Austroalpine and Southalpine domains, while they are totally lacking in the Helvetic domain. This distribution of HT-LP metamorphic assemblages supports the interpretation of an asymmetric rifting (e.g. Lardeaux \& Spalla, 1991; Marotta, Spalla \& Gosso, 2009).

\section{Conclusions}

We developed a 2D thermo-mechanical numerical model of passive rifting to investigate the evolution of the lithosphere of the Alps and the Northern Apennines during the transition from continental rifting to oceanic spreading of the Alpine Tethys. The model accounts for the crustal extension of a weak lower crust and mantle serpentinization, and results in symmetric rifting and denudation of the serpentinized lithospheric mantle.

A thinned continental crust (passive margin), an ocean-continent transition zone and an oceanic lithosphere characterize the final structure of the system.
The thickness of the passive margin decreases over time from $30 \mathrm{~km}$ to $18 \mathrm{~km}$ (hot model) or $22 \mathrm{~km}$ (cold model) at the model boundaries and to $5 \mathrm{~km}$ (hot model, hyperextended margin configuration) or $20 \mathrm{~km}$ (cold model) close to the ocean-continent transition zone.

The mantle serpentinization starts before the crustal break-up, and the denudation occurs before the oceanic spreading. In addition, a hot and weak lithosphere evolves to oceanization slower than a cold and strong lithosphere, with a comparable time interval after the onset of serpentinization.

Our results indicate that, if the estimated basin width is based simply on the coincidence between the continental crust break-up and the onset of the gabbros emplacement, the effective width of the basin domain will be underestimated. The mantle denudation starts several million years before partial melting, generating an ocean-continent transition zone from the passive continental margin to the oceanic lithosphere with a size ranging over $160-280 \mathrm{~km}$ in a magma-poor rift pre-Alpine configuration.

The thermo-barometric predictions with their modelled timing were compared with the natural data derived from continental and oceanic rocks from the Alps and Apennines of Permian-Jurassic age, and the predictions from the hot model, which also promotes the development of hyperextended Alpine margins, agree with natural data better.

Our results support the idea that the Tethyan rifting should begin in a perturbed continental lithosphere, likely ascribable to the previous Variscan subductioncollision, as widely supported by the occurrence of HP metamorphic relics in the different Alpine structural domains. In fact, if rifting developed in a stable lithosphere, Triassic-Jurassic HT-LP metamorphism is predicted together with gabbro-basalt production younger than $185 \mathrm{Ma}$ instead of the observed Permian-Triassic metamorphic and igneous records. Indeed, this has never been detected in the Alpine continental crust.

In addition, the distributions of the Permian-Triassic continental gabbros and the high-temperature metamorphism in the Austroalpine and Southalpine domains support the idea that it was asymmetric rifting in which the lithospheric signature of the Variscan subduction-collision can be a constraining inheritance for the successive geometry.

These ideas could be further confirmed by a new model that accounts for the previous history and the thermo-rheological consequences of Variscan Orogeny as initial configuration (as in Marotta, Spalla \& Gosso, 2009) and evolves though continental break-up and the successive oceanization as in the present study.

Acknowledgements. The research was supported by PRIN 2011 (2010AZR98L) (Birth and death of oceanic basins: geodynamic processes from rifting to continental collision in Mediterranean and circum-Mediterranean orogens). The authors thank T. Gerya and U. Ring for fruitful suggestions. We also thank the editor and the two anonymous reviewers for their thorough review. All figures were created using 
GMT plotting software (Wessel \& Smith, 2001) and Adobe Illustrator ${ }^{\circledR}$.

\section{References}

ARCAY, D., TRIC, E. \& DOIN, M. P. 2005. Numerical simulation of subduction zones. Effect of slab dehydration on the mantle wedge dynamics. Physics of the Earth and Planetary Interiors 149, 133-53.

Baumgartner, P. O., Bartolini, A., CARTer, E. S., Conti, M., Cortese, G., Danelian, T., De Wever, P., Dumitrica, P., DumitricA-Jud, R., GoricAN, S., Guex, J., Hull, D. M., Kito, N., Marcucci, M., Matsuoka, A., Murchey, B., O’Dogherty, L., SaVAry, J., VishneVsKaya, V., WidZ, D. \& YaO, A. 1995. Middle Jurassic to Early Cretaceous radiolarian biochronology of Tethys based on Unitary Associations. In Middle Jurassic to Lower Cretaceous Radiolaria of Tethys: Occurrences, Systematics, Biochronology (eds InterRad Jurassic-Cretaceous Working Group), pp. 23. Memoires de Geologie, Lausanne, Switzerland.

Beardsmore, G. R. \& CUlL, J. P. 2001. Crustal Heat Flow: A Guide to Measurement and Modelling. Cambridge: Cambridge University Press, $321 \mathrm{pp}$.

Beltrando, M., Rubatto, D. \& Manatschal, G. 2010. From passive margins to orogens: The link between ocean-continent transition zones and (ultra) highpressure metamorphism. Geology 38, 559-62.

Benciolini, L., Poli, M. E., Visona, D. \& Zanferrari, A. 2006. Looking inside Late Variscan tectonics: structural and metamorphic heterogeneity of the Eastern Southalpine basement (NE Italy). Geodinamica Acta 19, $17-32$.

Bertotti, G., Picotti, V., Bernoulli, D. \& CAStellarin, A. 1993. From rifting to drifting: tectonic evolution of the South-Alpine upper crust from the Triassic to the Early Cretaceous. Sedimentary Geology 86(1-2), 5376.

Best, M. G. \& Christiansen, E. H. 2001. Igneous Petrology. London: Blackwell Science, 458 pp.

Biagini, L., Bistacchi, A., Gosso, G., Magistroni, C., Rossetti, I., Spalla, M. I. \& TognONI, A. 1995. The II DK, HT mega-relic in the Sesia-Lanzo Zone: Late Variscan collision or Permo-Triassic rifting? In IOS International Ophiolite Symposium, Pavia, 18-23 September 1995, pp. 22.

Bigi, G., Castellarin, A., Coli, M., Dal Piaz, G.V., SARTORI, R., SCANDONE, P. \& VAI, G.B. 1990. Structural Model of Italy, sheets 1-2: CNR, Progetto Finalizzato Geodinamica.

Bill, M., O’Dogherty, L., Guex, J., Baumgartner, P. O. \& MASSON, H. 2001. Radiolarite ages in AlpineMediterranean ophiolites: Constraints on the oceanic spreading and the Tethys-Atlantic connection. Geological Society of America Bulletin 113(1), 129-43.

Bocquet, J., Delaloye, M., Hunziker, J. C. \& KRUMMENACHER, D. 1974. K-Ar and Rb-Sr dating of blue amphiboles, micas, and associated minerals from the Western Alps. Contributions to Mineralogy and Petrology 47(1), 7-26.

BoILlOT, G., BESLIER, M. O. \& GIRARDEAU, J. 1995. Nature, structure and evolution of the ocean-continent boundary: the lesson of the west Galicia margin (Spain). In Rifted Ocean-Continent Boundaries (eds. E. Banda, M. Torné \& M. Talwani), pp. 219-29. Dordrecht, Netherlands: Springer.
BOILlOT, G., GIRARDEAU, J. \& KORNPROBST, J. 1989. Rifting of the Galicia Margin: crustal thinning and emplacement of mantle rocks on the seafloor. In Proceedings of the Ocean Drilling Program, pp. 741-56. College Station, Texas, Scientific Results no. 103.

Bonin, B., BrÄndlein, P., Bussy, F., Desmons, J., Eggenberger, U., Finger, F., Graf, K., Marro, C., Mercolli, I., Oberhänsli, R., Ploquin, A., QuAdt, A. VON, RAUMER, J. VON, SCHALTEGGER, U., STEYRER, H. P. \& VisonÀ, D. 1993. In Pre-Mesozoic Geology in the Alps (eds. J. F. von Raumer \& F. Neubauer), pp. 327-44. Berlin, Heidelberg: Springer.

Boriani, A. \& Burlini, L. 1995. Carta Geologica della valle Cannobina Scala 1:25000. Milano: Dipartimento di Scienze della Terra "Ardito Desio" Università degli Studi di Milano.

Boriani, A., Colombo, A. \& Macera, P. 1985. Radiometric geochronology of Central Alps. Rendiconti della Società Italiana di Mineralogia e Petrologia 40, 139-86.

Borsi, S., Ferrara, G., Paganelli, L. \& Simboli, G. 1968. Isotopic age measurements of the M.Monzoni intrusive complex. Mineralogica et Petrographica Acta 14, 17183.

Bouffette, J., Lardeaux, J. M. \& Caron, J. M. 1993. Le passage des granulites aux éclogites dans les métapélites de l'unité de la Punta Muret (Massif Dora-Maira, Alpes occidentales). Comptes Rendus de l'Académie des Sciences 317, 1617-24.

Bousquet, R., Engi, M., Gosso, G., OBerhänsli, R., Berger, A., Spalla, M. I., Zucali, M. \& Goffè, B. 2004. Explanatory notes to the map: metamorphic structure of the Alps transition from the Western to the Central Alps. Mitteilungen der Österreichischen Mineralogischen Gesellschaft 149, 145-56.

Bozzo, E., CAmpi, S., CAPPONI, G. \& Giglia, G. 1992. The suture between the Alps and Apennines in the Ligurian sector based on geological and geomagnetic data. Tectonophysics 206(1-2), 159-69.

Brodie, K. H., Rex, D. \& RutTer, E. H. 1989. On the age of deep crustal extensional faulting in the Ivrea zone, Northern Italy. In Alpine Tectonics (eds R. G. Coward, M. P. Dietrich \& D. Park), pp. 203-10. Geological Society, London, Special Publication no. 45.

BRUNE, S. 2014. Evolution of stress and fault patterns in oblique rift systems: 3-D numerical lithospheric-scale experiments from rift to breakup. Geochemistry, Geophysics, Geosystems 15(8), 3392-415.

Brune, S. \& AUTIN, J. 2013. The rift to break-up evolution of the Gulf of Aden: Insights from 3D numerical lithospheric-scale modelling. Tectonophysics 607, 6579.

Brune, S., Heine, C., Pérez-Gussinyé, M. \& Sobolev, S. V. 2014. Rift migration explains continental margin asymmetry and crustal hyper-extension. Nature Communications 5, article no. 4014.

BUCK, W. R. 1991. Modes of continental lithospheric extension. Journal of Geophysical Research: Solid Earth 96(B12), 20161-78.

Buergi, A. \& KLOETZLi, U. 1990. New data on the evolutionary history of the Ivrea Zone (Northern Italy). Bulletin of the Swiss Association of Petroleum Geology and Engineering 56(130), 49-70.

Bussy, F., Venturini, G., HunZIKER, J. \& MARTINOTTI, G. 1998. U-Pb ages of magmatic rocks of the western Austroalpine Dent-Blanche-Sesia Unit. Schweizerische Mineralogische Und Petrographische Mitteilungen 78, $163-8$. 
CARminati, E. \& Doglioni, C. 2012. Alps vs. Apennines: The paradigm of a tectonically asymmetric Earth. EarthScience Reviews 112(1-2), 67-96.

CASSINIS, R. 2006. Reviewing pre-TRANSALP DFF models. Tectonophysics 414, 79-86.

ChALOT-PRAT, F. 2005. An undeformed ophiolite in the Alps: Field and geochemical evidence for a link between volcanism and shallow plate tectonic processes. In Plates, Plumes and Paradigms (eds G. R. Foulger, J. H. Natland, D. C. Presnall \& D. L. Anderson), pp. 750-80. Geological Society of America, Special Paper no. 388.

ChOPRA, P. N. \& Peterson, M. S. 1981. The experimental deformation of dunite. Tectonophysics 78, 453-73.

Clague, D. A. 1987. Hawaiian xenolith populations, magma supply rates, and development of magma chambers. Bulletin of Volcanology 49(4), 577-87.

Cloetingh, S., Burov, E., Matenco, L., Beekman, F., Roure, F. \& ZIEGler, P. A. 2013. The Moho in extensional tectonic settings: Insights from thermomechanical models. Tectonophysics 609, 558-604.

Colombo, A. \& TUNESI, A. 1999. Pre-Alpine metamorphism of the southern Alps. Schweizerische Mineralogische und Petrographische Mitteilungen 79, 63-77.

Compagnoni, R., Di Brozolo, F. R. \& SANDRONe, R. 1984. Kaersutite-bearing mylonitic gabbro from the Lanzoperidotite (western Italian Alps). Geologie en Mijnbouw 63(2), 189-96.

CORDEY, F. \& BAILlY, A. 2007. Alpine ocean seafloor spreading and onset of pelagic sedimentation: new radiolarian data from the Chenaillet-Montgenèvre ophiolite (French-Italian Alps). Geodinamica Acta, 20, 1318.

Corti, G., Bonini, M., Sokoutis, D., InNocenti, F., Manetti, P., Cloetingh, S. \& Mulugeta, G. 2004. Continental rift architecture and patterns of magma migration: A dynamic analysis based on centrifuge models. Tectonics 23(2), TC2012.

CostA, S. \& CABY, R. 2001. Evolution of the Ligurian Tethys in the Western Alps: $\mathrm{Sm} / \mathrm{Nd}$ and $\mathrm{U}-\mathrm{Pb}$ geochronology and rare-earth element geochemistry of the Montgenevre ophiolite (France). Chemical Geology 34(175), 449-66.

DAL PIAZ, G. V. 1993. Evolution of Austro-Alpine and Upper Penninic Basement in the Northwestern Alps from Variscan Convergence to Post-Variscan Extension. In Pre-Mesozoic Geology in the Alps (eds J. F. Raumer \& F. Neubauer), pp. 327-44. Berlin, Heidelberg: Springer.

DAL PIAZ, G. V. 2010. The Italian Alps: a journey across two centuries of Alpine geology. In The Geology of Italy: Tectonics and Life along Plate Margins (eds M. Beltrando, A. Peccerillo, M. Mattei, S. Conticelli \& C. Doglioni), pp. 1-108. Conder, Australia: Journal of the Virtual Explorer, Electronic Edition, 36.

Dal Piaz, G. V., De VeCchi, G. P. \& HunZiKeR, J. C. 1977. The Austroalpine layered gabbros of the Matterhorn and Mt. Collon-Dents de Bertol. Schweizerische Mineralogische und Petrographische Mitteilungen 57, 59-88.

DAl Piaz, G. V., LOMBARdo, B. \& Gosso, G. 1983. Metamorphic evolution of the Mt. Emilius klippe, Dent Blanche nappe, Western Alps. American Journal of Science 283A, 438-58.

De Capitani, L., Carnevale, M. \& Fumagalli, M. 2007. Gamma-ray spectroscopy determination of radioactive elements in late-Hercynian plutonic rocks of Val Biandino and Val Trompia (Lombardy, Italy). Periodico di Mineralogia 76(1), 25-39.

Del Moro, A. \& VisonÀ, D. 1982. The epiplutonic Hercynian Complex of Bressanone (Brixen, Eastern Alps,
Italy). Petrologic and radiometric data. Neues Jahrbuch fur Mineralogie - Abhandlungen 145, 66-85.

DESMONS, J. 1992. The Briançon basement (Pennine Western Alps): mineral composition and polymetamorphic evolution. Schweizerische Mineralogische und Petrographische Mitteilungen 72, 37-55.

Desmurs, L., Manatschal, G. \& Bernoulli, D. 2001. The Steinmann Trinity revisited: mantle exhumation and magmatism along an ocean-continent transition: the Platta nappe, eastern Switzerland. In Non-Volcanic Rifting of Continental Margins: A Comparison of Evidence from Land and Sea (eds. R.C.L. Wilson, R.B. Whitmarsh, B. Taylor \& N. Froitzheim), pp. 23566. Geological Society, London, Special Publication no. 187.

DI PAOlA, S. \& SPAlla, M. I. 2000. Contrasting tectonic records in pre-Alpine metabasites of the Southern Alps (lake Como, Italy). Journal of Geodynamics 30(1-2), 167-89.

Diella, V., Spalla, M. I. \& Tunesi, A. 1992. Contrasted thermo-mechanical evolutions in the South Alpine metamorphic basement of the Orobic Alps (Central Alps, Italy). Journal of Metamorphic Geology 10, 203-19.

Donatio, D., MARroni, M. \& RocChI, S. 2013. Serpentinization history in mantle section from a fossil slow-spreading ridge sequence/evidences from Pomaia quarry (Southern Tuscany, Italy). Ofioliti 38(1), 15-28.

Dubois, J. \& Diament, M. 1997. Géophysique. Paris: Masson, $205 \mathrm{pp}$.

England, P. C. \& Thompson, A. B. 1984. Pressuretemperature-time paths of regional metamorphism I. Heat transfer during the evolution of regions of thickened continental crust. Journal of Petrology 25(4), 894-928.

EscARTín, J., HiRTH, G. \& EvANS, B. 1997. Effects of serpentinization on the lithospheric strength and the style of normal faulting at slow-spreading ridges. Earth and Planetary Science Letters 151(3-4), 181-9.

Ewing, T., Hermann, J. \& Rubatto, D. 2013. The robustness of the Zr-in-rutile and Ti-in-zircon thermometers during high-temperature metamorphism (Ivrea-Verbano Zone, northern Italy). Contributions to Mineralogy and Petrology 165(4), 757-79.

Federico, L., Spagnolo, C., Crispini, L. \& Capponi, G. 2009. Fault-slip analysis in the metaophiolites of the Voltri Massif: constraints for the tectonic evolution at the Alps/Apennine boundary. Geological Journal 44(2), 225-40.

Ferry, J. M., Wing, B. A., Penniston-Dorland, S. C. \& RUMBLE, D. 2002. The direction of fluid flow during contact metamorphism of siliceous carbonate rocks: new data for the Monzoni and Predazzo aureoles, northern Italy, and a global review. Contributions to Mineralogy and Petrology 142(6), 679-99.

FontANA, E., PANSERI, M. \& TARTAROTTI, P. 2008. Oceanic relict textures in the Mount Avic Serpentinites, Western Alps. Ofioliti 33(2), 105-18.

Gaidies, F., AbarT, R., De CAPItAni, C., Schuster, R., Connolly, J. A. D. \& Reusser, E. 2006. Characterization of polymetamorphism in the Austroalpine basement east of the Tauern Window using garnet isopleth thermobarometry. Journal of Metamorphic Geology 24(6), 451-75.

Galli, A., Le Bayon, B., Schmidt, M. W., Burg, J.-P., CADDICK, M. J. \& REUSSER, E. 2011. Granulites and charnockites of the Gruf Complex: Evidence for Permian ultra-high temperature metamorphism in the Central Alps. Lithos 124(1-2), 17-45. 
Galli, A., Le Bayon, B., Schmidt, M. W., Burg, J.-P., Reusser, E., Sergeev, S. A. \& Larionov, A. 2012. $\mathrm{U}-\mathrm{Pb}$ zircon dating of the Gruf Complex: disclosing the late Variscan granulitic lower crust of Europe stranded in the Central Alps. Contributions to Mineralogy and Petrology 163(2), 353-78.

GALLIEN, F., ABART, R. \& WyHLIDAL, S. 2007. Contact metamorphism and selective metasomatism of the layered Bellerophon Formation in the eastern Monzoni contact aureole, northern Italy. Mineralogy and Petrology 91, $25-53$.

GARdien, V., Reusser, E. \& MARQuer, D. 1994. Pre-Alpine metamorphic evolution of the gneisses from the Valpelline series (Western Alps, Italy). Schweizerische Mineralogische und Petrographische Mitteilungen 74, 489502.

Garuti, G., BeA, F., Zaccarini, F. \& Montero, P. 2001. Age, geochemistry and petrogenesis of the ultramafic pipes in the Ivrea zone, NW Italy. Journal of Petrology 42(2), 433-57.

GERYA, T. V. \& STÖCKHERT, B. 2005. Two-dimensional numerical modeling of tectonic and metamorphic histories at active continental margins. International Journal of Earth Sciences 95(2), 250-74.

Giacomini, F., Messiga, B., Tribuzio, R. \& Braga, R. 1999. The Sondalo gabbroic complex and its country rocks: new geological and petrological data. Tübinger Geowissenschaftliche Arbeiten. Reihe A, Geologie, Palaeontologie, Stratigraphie, vol. 52, pp. 156.

GiLlcrist, R., COWARD, M. \& MugNiER, J. L. 1987. Structural inversion and its controls: examples from the Alpine foreland and the French-Alps. Geodinamica Acta 1, 5-34.

Goffé, B., Schwartz, S., Lardeaux, J. M. \& Bousquet, R. 2004. Explanatory notes to the map: metamorphic structure of the Alps Western and Ligurian Alps. Mitteilungen der Österreichischen Mineralogischen Gesellschaft 149, 125-44.

Gosso, G., Siletto, G. \& SPAlla, M. I. 1997. H-T/L-P metamorphism and structures in the South-Alpine basement near Lake Como, Orobic Alps; intracontinental imprints of the Permo-Triassic rifting. Ofioliti 22, 133-45.

GREGNANIN, A. 1980. Metamorphism and magmatism in the western Italian Tyrol. Rivista Italiana di Mineralogia e Petrologia 36, 49-64.

HAAS, R. 1985. Zur Metamorphose des Suedlichen Oetztalkristallins unter Besonderer Beruecksichtigung der Matscher Einheit (Vintschgau/Suedtirol). Ph.D. thesis, University of Innsbruck. Published thesis.

HABler, G. \& ThÖNI, M. 2001. Preservation of PermoTriassic low-pressure assemblages in the Cretaceous high-pressure metamorphic Saualpe crystalline basement (Eastern Alps, Austria). Journal of Metamorphic Geology 19, 679-97.

Handy, M. R., Franz, L., Heller, F., JanotT, B. \& ZURBriggen, R. 1999. Multistage accretion and exhumation of the continental crust (Ivrea crustal section, Italy and Switzerland). Tectonics 18(6), 1154-77.

HANDY, M. R. \& OBERHÄNSLI, R. 2004. Explanatory notes to the map: metamorphic structure of the Alps age map of the metamorphic structure of the Alps: tectonic interpretation and outstanding problem. Mitteilungen der Österreichischen Mineralogischen Gesellschaft 149, 20125.

Handy, M. R., Schmid, S. M., Bousquet, R., Kissling, E. \& Bernoulli, D. 2010. Reconciling plate-tectonic reconstructions of Alpine Tethys with the geological- geophysical record of spreading and subduction in the Alps. Earth-Science Reviews 102, 121-58.

HANDY, M. R. \& ZINGG, A. 1991. The tectonic and rheological evolution of an attenuated cross section of the continental crust: Ivrea crustal section, southern Alps, northwestern Italy and southern Switzerland. Geological Society of America Bulletin 103(2), 236-53.

Hansmann, W., Muntener, O. \& Hermann, J. 2001. U-Pb zircon geochronology of a tholeiitic intrusion and associated migmatites at a continental crust-mantle transition, Val Malenco, Italy. Schweizerische Mineralogische und Petrographische Mitteilungen 81(1), 239-56.

HÉBert, R., BeAudoin, G., Rochon, M. \& Gardien, V. 2008. Metamorphic evolution and oxygen isotope geochemistry of rift-precursor amphibolites from Hole 1067A ODP Leg 173 off West Iberian Galicia Bank rifted margin. Lithos 101, 162-76.

HenK, A., FranZ, L., Teufel, S. \& OnCKen, O. 1997. Magmatic underplating, extension, and crustal reequilibration: insights from a cross-section through the Ivrea Zone and Strona-Ceneri Zone, northern Italy. Journal of Geology 105(3), 367-77.

HERMANN, J. \& RUBATTO, D. 2003. Relating zircon and monazite domains to garnet growth zones: age and duration of granulite facies metamorphism in the Val Malenco lower crust. Journal of Metamorphic Geology 21(9), 833-52.

HoKE, L. 1990. The Altkristallin of the Kreuzeck Mountains, SE Tauern Window, Eastern Alps: Basement crust in a convergent plate boundary zone. Jahrbuch des Geologischen Bundesantall 133(1), 5-87.

HONDA, S. \& SAITO, M. 2003. Small-scale convection under the back-arc occurring in the low viscosity wedge. Earth and Planetary Science Letters 216, 703-15.

Huismans, R. S. \& Beaumont, C. 2011. Depth-dependent extension, two-stage breakup and cratonic underplating at rifted margins. Nature 473(7345), 74-78.

HuISMANS, R. S. \& BEAUMONT, C. 2014. Rifted continental margins: The case for depth-dependent extension. Earth and Planetary Science Letters 407, 148-62.

Huismans, R. S., Buiter, S. J. H. \& Beaumont, C. 2005. Effect of plastic-viscous layering and strain softening on mode selection during lithospheric extension. Journal of Geophysical Research: Solid Earth 110(B2), 1-17.

HunZIKER, J. C., DeSMON, J. \& HuRforD, A. J. 1992. Thirtytwo years of geochronological work in the Central and Western Alps: a review of seven maps. Mémoires de Géologie, Lausanne 13, 1-59.

HunZIKER, J. C. \& ZINGG, A. 1980. Lower Paleozoic amphibolite to granulite facies metamorphism in the Ivrea Zone (southern Alps, northern Italy). Schweizerische Mineralogische und Petrographische Mitteilungen 60, 181-213.

KaczmareK, M.-A., Müntener, O. \& Rubatto, D. 2008. Trace element chemistry and U-Pb dating of zircons from oceanic gabbros and their relationship with whole rock composition (Lanzo, Italian Alps). Contributions to Mineralogy and Petrology 155(3), 295-312.

Klötzli, U. S., SinigoI, S., Quick, J. E., Demarchi, G., TASSINARI, C. C. G., SATO, K. \& GÜNES, Z. 2014. Duration of igneous activity in the Sesia Magmatic System and implications for high-temperature metamorphism in the Ivrea-Verbano deep crust. Lithos 206, 19-33.

LAGABRIElle, Y. \& CANNAT, M. 1990. Alpine Jurassic ophiolites resemble the modern central Atlantic basement. Geology 18(4), 319-22.

Lagabrielle, Y., Fudral, S. \& Kienast, J.-R. 1989. La couverture océanique des ultrabasites de Lanzo (Alpes 
occidentales): arguments lithostratigraphiques et pétrologiques. Geodinamica Acta 4(1), 43-55.

LANGONE, A. \& TiePOLO, M. 2015. U-Th-Pb 'multi-phase' approach to the study of crystalline basement: application to the northernmost sector of the Ivrea-Verbano Zone (Alps). Periodico di Mineralogia 84(3B), 633-55.

LARDEAUX, J. M. 1981. Evolution Tectono-metamorphique de la Zone Nord du Massif de Sesia-Lanzo (Alpes Occidentales): Un Exemple d'Éclogitisation de Croute Continentale. Ph.D. thesis, University of Paris VI. Published thesis.

LARDEAUX, J.-M. 2014. Deciphering orogeny: a metamorphic perspective. Examples from European Alpine and Variscan belts: Part I: Alpine metamorphism in the western Alps. A review. Bulletin de la Societe Geologique de France 185(2), 93-114.

LARDEAUX, J. M. \& Spalla, M. I. 1991. From granulites to eclogites in the Sesia zone (Italian Western Alps): a record of the opening and closure of the Piedmont Ocean. Journal of Metamorphic Geology 9(1), 35-59.

LAVIER, L. L. \& MANATSCHAL, G. 2006. A mechanism to thin the continental lithosphere at magma-poor margins. Nature 440(7082), 324-8.

Li, X.-H., Faure, M., Lin, W. \& Manatschal, G. 2013. New isotopic constraints on age and magma genesis of an embryonic oceanic crust: the Chenaillet Ophiolite in the Western Alps. Lithos 160-161, 283-91.

Li, X.-H., FAure, M., Rossi, P., Lin, W. \& LAhOndère, D. 2015. Age of Alpine Corsica ophiolites revisited: Insights from in situ zircon $\mathrm{U}-\mathrm{Pb}$ age and $\mathrm{O}-\mathrm{Hf}$ isotopes. Lithos 220-223, 179-90.

LIAO, J. \& GERYA, T. 2015. From continental rifting to seafloor spreading: Insight from $3 \mathrm{D}$ thermo-mechanical modeling. Gondwana Research 28(4), 1329-43.

Lu, M., Hofmann, A. W., Mazzucchelli, M. \& Rivalenti, G. 1997. The mafic-ultramafic complex near Finero (Ivrea-Verbano Zone), II. Geochronology and isotope geochemistry. Chemical Geology 140(3-4), 223-35.

Malatesta, C., Crispini, L., Federico, L., CAPPoni, G. \& SCAMBELLURI, M. 2012. The exhumation of high pressure ophiolites (Voltri Massif, Western Alps): Insights from structural and petrologic data on metagabbro bodies. Tectonophysics 568-569, 102-23.

Malatesta, C., Gerya, T., Crispini, L., Federico, L. \& CAPPONI, G. 2013. Oblique subduction modelling indicates along-trench tectonic transport of sediments. Nature Communications 4, 1-6.

MANATSCHAL, G. 2004. New models for evolution of magma-poor rifted margins based on a review of data and concepts from West Iberia and the Alps. International Journal of Earth Sciences 93(3), 432-66.

Manatschal, G., LAVIER, L. \& Chenin, P. 2015. The role of inheritance in structuring hyperextended rift systems: Some considerations based on observations and numerical modeling. Gondwana Research 27(1), 140-64.

Manatschal, G. \& Müntener, O. 2009. A type sequence across an ancient magma-poor ocean-continent transition: the example of the western Alpine Tethys ophiolites. Tectonophysics 473(1-2), 4-19.

Manatschal, G., SAuter, D., Karpoff, A. M., Masini, E., Mohn, G. \& LagabrielLe, Y. 2011. The Chenaillet Ophiolite in the French/Italian Alps: An ancient analogue for an Oceanic Core Complex? Lithos 124(3-4), 169-84.

ManzotTi, P. \& Zucali, M. 2013. The pre-Alpine tectonic history of the Austroalpine continental basement in the Valpelline unit (Western Italian Alps). Geological Magazine 150(1), 153-72.
Marotta, A. M. \& Spalla, M. I. 2007. Permian-Triassic high thermal regime in the Alps: Result of late Variscan collapse or continental rifting? Validation by numerical modeling. Tectonics 26, 1-27.

Marotta, A. M., Spalla, M. I. \& Gosso, G. 2009. Upper and lower crustal evolution during lithospheric extension: numerical modelling and natural footprints from the European Alps. In Extending a Continent: Architecture, Rheology and Heat Budget (eds U. Ring \& B. Wernicke), pp. 33-72. The Geological Society, London, Special Publication no. 321.

Marotta, A. M., Spelta, E. \& Rizzetto, C. 2006. Gravity signature of crustal subduction inferred from numerical modelling. Geophysical Journal International 166, 923-38.

Marroni, M., Molli, G., Montanini, A. \& Tribuzio, R. 1998. The association of continental crust rocks with ophiolites in the Northern Apennines (Italy): implications for the continent-ocean transition in the Western Tethys. Tectonophysics 292(1-2), 43-66.

MARRONI, M. \& PANDOLFI, L. 2007. The architecture of an incipient oceanic basin: a tentative reconstruction of the Jurassic Liguria-Piemonte basin along the Northern Apennines-Alpine Corsica transect. International Journal of Earth Sciences 96(6), 1059-78.

MARRONI, M. \& TRIBUZIO, R. 1996. Gabbro-derived granulites from external liguride units (northern Apennine, Italy): implications for the rifting processes in the western Tethys. Geologische Rundschau 85(2), 239-49.

Martin, S., Tartarotti, P. \& Dal Piaz Giorgio, V. 1994. The Mesozoic ophiolites of the Alps: a review. OGS/Bollettino di Geofisica Teorica e Applicata 36(141-144), 175-220.

Mayer, A., Mezger, K. \& SinigoI, S. 2000. New Sm$\mathrm{Nd}$ ages for the Ivrea-Verbano Zone, Sesia and Sessera valleys (Northern Italy). Journal of Geodynamics 30(12), 147-66.

Mevel, C., CaBy, R. \& Kienast, J.-R. 1978. Amphibolite facies conditions in the oceanic crust: example of amphibolitized flaser-gabbro and amphibolites from the Chenaillet ophiolite massif (Hautes Alpes, France). Earth and Planetary Science Letters 39(1), 98-108.

MiLlER, C. \& THÖNI, M. 1997. Eo-alpine eclogitisation of Permian MORB-type gabbros in the Koralpe (Eastern Alps, Austria): new geochronological, geochemical and petrological data. Chemical Geology 137(3-4), 283310.

Miller, C., ThÖNI, M., Goessler, W. \& TESSADRI, R. 2011. Origin and age of the Eisenkappel gabbro to granite suite (Carinthia, SE Austrian Alps). Lithos 125(1-2), 434-48.

Mohn, G., Manatschal, G., Beltrando, M., Masini, E. \& KUSZNIR, N. 2012. Necking of continental crust in magma-poor rifted margins: Evidence from the fossil Alpine Tethys margins. Tectonics 31(1), TC1012.

MonjoIE, P. 2004. The Mont Collon mafic complex (Austroalpine Dent Blanche nappe): Permian evolution of the Western European mantle. Ph.D. thesis. Université de Lausanne. Published thesis.

Monjoie, P., Bussy, F., Lapierre, H. \& Pfeifer, H.-R. 2005. Modeling of in-situ crystallization processes in the Permian mafic layered intrusion of Mont Collon (Dent Blanche nappe, western Alps). Lithos 83(3-4), 317-46.

Montanini, A., TribuZio, R. \& AnCZKiewicz, R. 2006. Exhumation history of a garnet pyroxenite-bearing mantle section from a continent-ocean transition (northern Apennine Ophiolites, Italy). Journal of Petrology 47(10), 1943-71. 
Mundil, R., Brack, P. \& LAurenzi, M. A. 1996. High resolution $\mathrm{U}-\mathrm{Pb}$ single-zircon age determinations: new constraints on the timing of Middle Triassic magmatism in the Southern Alps. In 78 a Riunione Estiva, Geologia delle Dolomiti, pp. 1, Società Geologica Italiana. San Cassiano, 16-18 September 1996.

Muntener, O. \& HeRMAnN, J. 2001. The role of lower crust and continental upper mantle during formation of non-volcanic passive margins: evidence from the Alps. In Non-Volcanic Rifting of Continental Margins: A Comparison of Evidence from Land and Sea (eds. R.C.L. Wilson, R.B. Whitmarsh, B. Taylor \& N. Froitzheim), pp. 267-88. Geological Society, London, Special Publication no. 187.

Muntener, O., HermanN, J. \& Trommsdorf, V. 2000. Cooling history and exhumation of lower-crustal granulite and upper mantle (Malenco, eastern Central Alps). Journal of Petrology 41(2), 175-200.

NAGEL, T. J. \& BUCK, W. R. 2004. Symmetric alternative to asymmetric rifting models. Geology 32(11), 937-40.

Nagy, G., Draganits, E., Demény, A., Pantó, G. \& ÁRKAI, P. 2002. Genesis and transformations of monazite, florencite and rhabdophane during medium grade metamorphism: examples from the Sopron Hills, Eastern Alps. Chemical Geology 191(1-3), 25-46.

NALIBOFF, J. \& BUITER, S. J. H. 2015. Rift reactivation and migration during multiphase extension. Earth and Planetary Science Letters 421, 58-67.

NicoT, E. 1977. Les roches meso and catazonales de la Valpelline (nappe de la Dent Blanche; Alpes Italiennes). $\mathrm{Ph}$.D. thesis. Université Pierre et Marie Curie, Paris VI. Published thesis.

OHNEnStetter, M., OHNEnStetTer, D., VidAl, P., Cornichet, J., Hermitte, D. \& MACE, J. 1981. Crystallization and age of zircon from Corsican ophiolitic albitites: consequences for oceanic expansion in Jurassic times. Earth and Planetary Science Letters 54(3), 397-408.

PenNACCHIONI, G. \& CeSARE, B. 1997. Ductile-brittle transition in pre-Alpine amphibolite facies mylonites during evolution from water-present to water-deficient conditions (Mont Mary nappe, Italian Western Alps). Journal of Metamorphic Geology 15(6), 777-91.

Peressini, G., QUick, J. E., Sinigoi, S., HofmanN, A. W. \& FANNING, M. 2007. Duration of a large mafic intrusion and heat transfer in the lower crust: a SHRIMP U-Pb zircon study in the Ivrea-Verbano Zone (western Alps, Italy). Journal of Petrology 48(6), 1185-218.

Pérez-Gussinyé, M., Morgan, J. P., Reston, T. J. \& RANERO, C. R. 2006. The rift to drift transition at nonvolcanic margins: Insights from numerical modelling. Earth and Planetary Science Letters 244(1-2), 458-73.

PÉreZ-GussinYé, M., Reston, T. J. \& PhipPS MORgan, J. 2001. Serpentinization and magmatism during extension at non-volcanic margins: the effect of initial lithospheric structure. In Non-Volcanic Rifting of Continental Margins: A Comparison of Evidence from Land and Sea (eds. R.C.L. Wilson, R.B. Whitmarsh, B. Taylor \& N. Froitzheim), pp. 551-76. Geological Society, London, Special Publication no. 187.

PicCARdo, G. B. \& GUARNIERI, L. 2010. Alpine peridotites from the Ligurian Tethys: an updated critical review. International Geology Review 52(10-12), 1138-59.

Piccardo, G. B., Padovano, M. \& GuARnieri, L. 2014. The Ligurian Tethys: Mantle processes and geodynamics. Earth-Science Reviews 138, 409-34.

PIN, C. 1986. Datation U-Pb sur zircon à $285 \mathrm{Ma}$ du complexe gabbro dioritique du Val Sesia - Val Mastallone et age tardi hercynien du métamorphisme granulitique de la zone Ivrea-Verbano (Italie). Compte Rendu Academie des Sciences de Paris 303, 827-30.

PINARELLI, L. \& BorIANI, A. 2007. Tracing metamorphism, magmatism and tectonics in the southern Alps (Italy): constraints from $\mathrm{Rb}-\mathrm{Sr}$ and $\mathrm{Pb}-\mathrm{Pb}$ geochronology, and isotope geochemistry. Periodico di Mineralogia 76, 524.

Pinto, V. H. G., Manatschal, G., Karpoff, A. M. \& ViAnA, A. 2015. Tracing mantle-reacted fluids in magma-poor rifted margins: The example of Alpine Tethyan rifted margins. Geochemistry, Geophysics, Geosystems 16(9), 3271-308.

PLATT, J. P. 1986. Dynamic of orogenic wedges and the uplift of high-pressure metamorphic rocks. Geological Society of America Bulletin 97, 1037-1053.

Pognante, U., Rösli, U. \& ToscANI, L. 1985. Petrology of ultramafic and mafic rocks from the Lanzo peridotite body (Western Alps). Lithos 18, 201-14.

Polino, R., Dal Piaz, G. V. \& Gosso, G. 1990. Tectonic erosion at the Adria margin and accretionary processes for the Cretaceous orogeny of the Alps. Mémoires de la Societé Géologique de France 156, 345-67.

POVODEN, E., HORACEK, M. \& ABART, 2002. Contact metamorphism of siliceous dolomite and impure limestones from the Werfen formation in the eastern Monzoni contact aureole. Mineralogy and Petrology 76, 99-120.

Quick, J. E., SinigoI, S., Negrini, L., Demarchi, G. \& MAYER, A. 1992. Synmagmatic deformation in the underplated igneous complex of the Ivrea-Verbano zone. Geology 20(7), 613-6.

Quick, J. E., Sinigoi, S., Snoke, A. W., Kalakay, T. J., MAYER, A. \& PERESSINI, G. 2002. Geologic map of the southern Ivrea-Verbano zone, northwestern Italy. In Geologic Investigation Series Map I-2776 and booklet, pp. 22. Reston, Virginia: US Geological Survey, US Government Printing Office.

RAMPONE, E. 2002. Mantle dynamics during PermoMesozoic extension of the Europe-Adria lithosphere: insights from the Ligurian ophiolites. Periodico di Mineralogia 73, 215-30.

Rampone, E., Borghini, G., Romairone, A., Abouchami, W., Class, C. \& Goldstein, S. L. 2014. Sm-Nd geochronology of the Erro-Tobbio gabbros (Ligurian Alps, Italy): Insights into the evolution of the Alpine Tethys. Lithos 205, 236-46.

RAMPONE, E., HOFMANN, A. W. \& RACZEK, I. 2009. Isotopic equilibrium between mantle peridotite and melt: Evidence from the Corsica ophiolite. Earth and Planetary Science Letters 288(3-4), 601-10.

RANALli, G. \& MurPhy, D. C. 1987. Rheological stratification of the lithosphere. Tectonophysics 132(4), 281-95.

REBAY, G., RICCARDI, M. P. \& SPALLA, M. I. 2015. Fluid rock interactions as recorded by Cl-rich amphiboles from continental and oceanic crust of italian orogenic belts. Periodico di Mineralogia 84(3B), 751-77.

REBAY, G. \& SPALLA, M. I. 2001. Emplacement at granulite facies conditions of the Sesia-Lanzo metagabbros: an early record of Permian rifting? Lithos 58(3-4), 85-104.

Reston, T. J. \& MoRGan, J. P. 2004. Continental geotherm and the evolution of rifted margins. Geology 32(2), 1336.

Riccardi, M. P., Tribuzio, R. \& Caucia, F. 1994. Amphibole evolution in the metagabbros from East Ligurian ophiolites (Northern Apennines, Italy): Constraints on the ocean-floor metamorphism. Memorie della Società Geologica Italiana 48, 203-8. 
Roda, M., Marotta, A. M. \& Spalla, M. I. 2010. Numerical simulations of an ocean-continent convergent system: Influence of subduction geometry and mantle wedge hydration on crustal recycling. Geochemistry, Geophysics, Geosystems 11(5), 1-21.

RodA, M., Spalla, M. I. \& MAROTTA, A. M. 2012. Integration of natural data within a numerical model of ablative subduction: a possible interpretation for the Alpine dynamics of the Austroalpine crust. Journal of Metamorphic Geology 30(9), 973-996.

Rogers, N., BLAKE, S. K. B., WidDOWSON, M., PARKINSON, I. \& HARRIS, N. 2008. An Introduction to Our Dynamic Planet. New York: Cambridge University Press, 398 pp.

Rossi, P., COCHERIE, A., LAHONDĖRE, D. \& FANNING, C. M. 2002. La marge Européenne de la Téthys Jurassique en Corse: datation de trondhjémites de Balagne et indices de croûte continentale sous le domaine Balano-Ligure. Comptes Rendus Geoscience 334(5), 313-22.

Rossi, P., LAhondère, J.-C., Cocherie, A., CABAllero, Y. \& FÉRAUD, J. 2012. Notice explicative, Carte geol. France (1/50 000), feuille Bastelica (1118). Edition du Bureau de Recherches Géologiques et Minières, Orléans, $134 \mathrm{pp}$.

Rottura, A., Bargossi, G. M., Caggianelli, A., Del Moro, A., VisonÀ, D. \& Tranne, C. A. 1998. Origin and significance of the Permian high-K calc-alkaline magmatism in the central-eastern Southern Alps, Italy. Lithos 45(1-4), 329-48.

Rubatto, D., Regis, D., Hermann, J., Boston, K., Engi, M., Beltrando, M. \& McAlpine, S. R. B. 2011. Yo-yo subduction recorded by accessory minerals in the Italian Western Alps. Nature Geoscience 4(5), 338-42.

RYBACH, L. 1988. Determination of heat production rate. In Handbook of Terrestrial Heat-Flow Density Determination (eds R. Haenel, L. Stegena \& L. Rybach), pp. 125142. Solid Earth Sciences Library, Kluwer Academic Publishers.

SAnders, C. A. E., Bertotti, G. \& Tommasini, S. 1996. Triassic pegmatites in the Mesoizoic middle crust of the Southern Alps (Italy): fluid inclusions, radiometric dating and tectonic implications. Eclogae Geologicae Helvetiae 89(1), 505-25.

SANDIFORD, M. \& Powell, R. 1986. Deep crustal metamorphism during crustal extension: modern and ancient examples. Earth and Planetary Science Letters 79, 1518.

SCHMID, S. M., FÜGENSCHUH, B., KISSLING, E. \& SCHUSTER, R. 2004. Tectonic map and overall architecture of the Alpine orogen. Eclogae Geologicae Helvetiae 97(1), 93-117.

SCHUSTER, R. \& FRANK, W. 1999. Metamorphic evolution of the Austroalpine units east of the Tauern Window: indications for Jurassic strike slip tectonics. Mitteilungen der Gesellschaft der Geologie und Bergbaustudenten in Österreich 42, 37-58.

Schuster, R., Scharbert, S., Abart, R. \& Frank, W. 2001. Permo-Triassic extension and related HT/LP metamorphism in the Austroalpine-Southalpine realm. Mitteilungen der Gesellschaft der Geologie und Bergbaustudenten in Österreich 45, 111-41.

Schuster, R. \& StÜWE, K. 2008. Permian metamorphic event in the Alps. Geology 36, 603-6.

SERANNE, M. 1999. The Gulf of Lion continental margin (NW Mediterranean) revisited by IBS: an overview. In The Mediterranean Basins: Tertiary Extension within the Alpine Orogen (eds. B. Durand, L. Jolivet, F. Horvath \& M. Seranne), pp. 15-36. Geological Society, London, Special Publication no. 156.
SILLS, J. D. 1984. Granulite facies metamorphism in the Ivrea Zone, NW Italy. Schweizerische Mineralogische und Petrographische Mitteilungen 64, 169-91.

SmYe, A. J. \& STOCKLI, D. F. 2014. Rutile U-Pb age depth profiling: A continuous record of lithospheric thermal evolution. Earth and Planetary Science Letters $\mathbf{4 0 8}$, 171-82.

Spalla, M. I., LardeauX, J. M., Dal Piaz, G. V. \& Gosso, G. 1991. Metamorphisme et tectonique a la marge externe de la zone Sesia-Lanzo (Alpes occidentales). Memorie di Scienze Geologiche 43, 361-9.

Spalla, M. I., LardeauX, J. M., Dal Piaz, G. V., Gosso, G. \& MessigA, B. 1996. Tectonic significance of the Alpine eclogites. Journal of Geodynamics 21(3), 257-85.

Spalla, M. I., Messiga, B. \& Gosso, G. 1995. LT-Alpine overprint on the HT rifting-related metamorphism in the steep belt of the Languard-Campo Nappe. The Cima Rovaia and Scisti del Tonale Units represent two different extents of Alpine re-equilibration. In International Ophiolite Symposium, Pavia, 18-23 September 1995, pp. 148.

Spalla, M. I., Zanoni, D., Gosso, G. \& ZuCALI, M. 2009 Deciphering the geologic memory of a Permian conglomerate of the Southern Alps by pebble P-T estimates. International Journal of Earth Sciences 98(1), 203-26.

Spalla, M. I., ZanONI, D., MarotTA, A. M., Rebay, G., RodA, M., Zucali, M. \& Gosso, G. 2014. The transition from Variscan collision to continental break-up in the Alps: insights from the comparison between natural data and numerical model predictions. In The Variscan Orogeny: Extent, Timescale and the Formation of the European Crust (eds K. Schulmann, J. R. Martínez Catalán, J. M. Lardeaux, V. Janoušek \& G. Oggiano), pp. 363-400. Geological Society, London, Special Publication no. 405.

SPEAR, F. S. \& PEACOCK, S. M. 1989. Metamorphic PressureTemperature-Time Paths. Washington, DC: American Geophysical Union, 102 pp.

Spicker, G. \& Huckenholz, H. G. 1986. Petrography and geochemistry of the Monzoni Intrusion, northern Italy. Fortschritte der Mineralogie, Beiheft 64(1), 172.

Spiess, R., Cesare, B., Mazzoli, C., SAssi, R. \& SASSI, F. P. 2010. The crystalline basement of the Adria microplate in the eastern Alps: a review of the palaeostructural evolution from the Neoproterozoic to the Cenozoic. Rendiconti Lincei Scienze Fisiche Naturali 21, 31-50.

Stahle, V., Frenzel, G., Hess, J. C., SAupe, F., Schmidt, S. T. \& SCHNEIDER, W. 2001. Permian metabasalt and Triassic alkaline dykes in the northern Ivrea zone: clues to the post-Variscan geodynamic evolution of the Southern Alps. Schweizerische Mineralogische Und Petrographische Mitteilungen 81, 1-21.

Stampfli, G., Mosar, J., Marquer, D., Marchant, R., BAUDIN, T. \& BOREL, G. 1998. Subduction and obduction processes in the Swiss Alps. Tectonophysics 296(12), 159-204.

StÖCKHERT, B. \& GerYA, T. V. 2005. Pre-collisional high pressure metamorphism and nappe tectonics at active continental margins: a numerical simulation. Terra Nova 17, 102-10.

STÖCKHERT, B. 1987. Das Uttenheimer Pegmatitfeld (Ostalpines Altkristallin, Suedtirol) Genese und alpine Ueberpraegung. Erlanger Geologische Abhandlungen 114, 83-106.

TENCZER, V., Powell, R. \& STUwe, K. 2006. Evolution of $\mathrm{H} 2 \mathrm{O}$ content in a polymetamorphic terrane: the Plattengneiss Shear Zone (Koralpe, Austria). Journal of Metamorphic Geology 24(4), 281-95. 
ThOMPSON, A. B. 1981. The pressure-temperature (P,T) plane viewed by geophysicists and petrologists. Terra Cognita 1, 11-20.

Thompson, A. B. \& England, P. C. 1984. Pressuretemperature-time paths of regional metamorphism II. Their inference and interpretation using mineral assemblages in metamorphic rocks. Journal of Petrology 25(4), 929-55.

THÖNI, M. \& JAGOUTZ, E. 1992. Some new aspects of dating eclogites in orogenic belts: $\mathrm{Sm}-\mathrm{Nd}, \mathrm{Rb}-\mathrm{Sr}$, and $\mathrm{Pb}-\mathrm{Pb}$ isotopic results from the Austroalpine Saualpe and Koralpe type-locality (Carinthia/Styria, southeastern Austria). Geochimica et Cosmochimica Acta 56(1), 347-68.

ThÖNI, M. \& Miller, C. 2000. Permo-Triassic pegmatites in the eo-Alpine eclogite-facies Koralpe complex, Austria: age and magma source constraints from mineral chemical, $\mathrm{Rb}-\mathrm{Sr}$ and $\mathrm{Sm}-\mathrm{Nd}$ isotope data. Schweizerische Mineralogische und Petrographische Mitteilungen 80(2), 169-86.

ThÖNI, M. \& Miller, C. 2009. The 'Permian event' in the Eastern European Alps: $\mathrm{Sm}-\mathrm{Nd}$ and $\mathrm{P}-\mathrm{T}$ data recorded by multi-stage garnet from the Plankogel unit. Chemical Geology 260(1-2), 20-36.

ThÖNI, M., Miller, C., Blichert-Toft, J., Whitehouse, M. J., Konzett, J. \& ZAnetti, A. 2008. Timing of high-pressure metamorphism and exhumation of the eclogite type-locality (Kupplerbrunn-Prickler Halt, Saualpe, south-eastern Austria): constraints from correlations of the $\mathrm{Sm}-\mathrm{Nd}, \mathrm{Lu}-\mathrm{Hf}, \mathrm{U}-\mathrm{Pb}$ and $\mathrm{Rb}-\mathrm{Sr}$ isotopic systems. Journal of Metamorphic Geology 26(5), 56181.

Thöni, M., Mottana, A., Delitala, M. C., De Capitani, L. \& LibORIO, G. 1992. The Val Biandino composite pluton: a Late Hercynian intrusion into the South Alpine metamorphic basement of the Alps (Italy). Neues Jahrbuch fur Mineralogie - Abhandlungen 12, 545-54.

TRIBUZIO, R., Riccardi, M. P. \& Messiga, B. 1997. Amphibolitization of $\mathrm{Mg}$ - and $\mathrm{Fe}$-rich gabbroic dykes within mantle-derived serpentinites from Northern Apennine ophiolites: Evidence for high-temperature hydration of the oceanic lithosphere. Ofioliti 22(1), 71-80.

Tribuzio, R., Riccardi, M. P. \& OtTOLINI, L. 1995. Trace element redistribution in high-temperature deformed gabbros from East Ligurian ophiolites (Northern Apennines, Italy): constraints on the origin of syndeformation fluids. Journal of Metamorphic Geology 13(3), 367-77.

Tribuzio, R., Thirwall, M. F. \& Messiga, B. 1999. Petrology, mineral and isotope geochemistry of the Sondalo gabbroic complex (Central Alps, Northern Italy): implications for the origin of post-Variscan magmatism. Contributions to Mineralogy and Petrology 136, 48-62.

TRIBUZIO, R., THIRWALL, M. F. \& VANNUCCI, R. 2004. Origin of the gabbro-peridotite association from the Northern Apennine Ophiolites (Italy). Journal of Petrology 45(6), 1109-24.

Turco, E., Macchiavelli, C., Mazzoli, S., Schettino, A. \& PIERANTONI, P. P. 2012. Kinematic evolution of Alpine Corsica in the framework of Mediterranean mountain belts. Tectonophysics 579, 193-206.

Turner, S. P., George, R. M. M., Evans, P. J., Hawkesworth, C. J. \& Zellmer, G. F. 2000. Timescales of magma formation, ascent and storage beneath subduction-zone volcanoes. Philosophical Transactions of the Royal Society A: Mathematical, Physical and Engineering Sciences 358(1770), 1443-64.

Van AvendonK, H. J. A., LaVier, L. L., Shillington, D. J. \& MANATSCHAL, G. 2009. Extension of continental crust at the margin of the eastern Grand Banks, Newfoundland. Tectonophysics 468(1-4), 131-48.

Vavra, G., Gebauer, D., Schmid, R. \& Compston, W. 1996. Multiple zircon growth and recrystallization during polyphase Late Carboniferous to Triassic metamorphism in granulites of the Ivrea Zone (Southern Alps): an ion microprobe (SHRIMP) study. Contributions to Mineralogy and Petrology 122(4), 337-58.

VAVRA, G., SCHMID, R. \& GEBAUER, D. 1999. Internal morphology, habit and $\mathrm{U}-\mathrm{Th}-\mathrm{Pb}$ microanalysis of amphibolite-to-granulite facies zircons: geochronology of the Ivrea Zone (Southern Alps). Contributions to Mineralogy and Petrology 134(4), 380-404.

VISONÀ, D. 1995. Polybaric evolution of calc-alkaline magmas: the dioritic belt of the Bressanone-Chiusa igneous complex (NE Italy). Memorie di Scienze Geologiche 47, 111-24.

VISONÀ, D. 1997. The Predazzo multipulse intrusive body (Western Dolomites, Italy). Field and mineralogical studies. Memorie di Scienze Geologiche 49, 117-25.

VisonÀ, D., Fioretti, A. M., Poli, M. E., ZANFERrari, A. \& FANNING, M. 2007. U-Pb SHRIMP zircon dating of andesite from the Dolomite area (NE Italy): geochronological evidence for the early onset of Permian Volcanism in the eastern part of the southern Alps. Swiss Journal of Geosciences 100(2), 313-24.

VON RAUMER, J. F., BusSY, F., SCHALTEGger, U., SCHUlz, B. \& StAmpfli, G. M. 2013. Pre-Mesozoic Alpine basements: Their place in the European Paleozoic framework. Geological Society of America Bulletin 125(1-2), 89-108.

Voshage, H., HunziKer, J. C., HoffmanN, A. W. \& ZINGG, A. 1987. A $\mathrm{Nd}$ and $\mathrm{Sr}$ isotopic study of Ivrea zone, Southern Alps, N-Italy. Contributions to Mineralogy and Petrology 97, 31-42.

Vuichard, J. P. 1987. Conditions P-T du métamorphisme anté-alpin dans la seconde zone diorito-kinzigitique (Zone Sesia-Lanzo, Alpes occidentales). Schweizerische Mineralogische und Petrographische Mitteilungen 67, 257-71.

WeSSEL, P. \& SMITH, W. M. F. 2001. New improved version of generic mapping tools released. EOS Transactions of the American Geophysical Union 79, 579.

Whitmarsh, R. B., Manatschal, G. \& Minshull, T. A. 2001. Evolution of magma-poor continental margins from rifting to seafloor spreading. Nature 413(6852), $150-4$.

Wogelius, R. A. \& Finley, B. G. 1989. Subsolidus emplacement history of the Lanzo massif, northern Italy. Geology 17(11), 995-9.

Zanoni, D., Spalla, M. I. \& Gosso, G. 2010. Vestiges of lost tectonic units in conglomerate pebbles? A test in Permian sequences of the Southalpine Orobic Alps. Geological Magazine 147(1), 98-122.

ZUCALI, M. 2001. La correlazione nei terreni metamorfici: due esempi dall'Austroalpino occidentale (Zona SesiaLanzo) e centrale (Falda Languard-Campo/ Serie del Tonale). Ph.D. thesis, Università degli Studi di Milano. Published thesis. 